\title{
A UNIQUENESS CRITERION FOR MEASURE-VALUED SOLUTIONS OF SCALAR HYPERBOLIC CONSERVATION LAWS
}

\author{
MICHIEL BERTSCH, FLAVIA SMARRAZZO, ANDREA TERRACINA, \\ AND ALBERTO TESEI
}

\begin{abstract}
We prove existence and uniqueness of Radon measure-valued solutions of the Cauchy problem$$
\begin{cases}u_{t}+[\varphi(u)]_{x}=0 & \text { in } \mathbb{R} \times(0, T) \\ u=u_{0} \geq 0 & \text { in } \mathbb{R} \times\{0\}\end{cases}
$$

where $u_{0}$ a positive Radon measure whose singular part is a finite superposition of Dirac masses, and $\varphi \in C^{2}([0, \infty))$ is bounded. The novelty of the paper is the introduction of a compatibility condition which, combined with standard entropy conditions, guarantees uniqueness.
\end{abstract}

\section{IntRoduction}

1.1. Statement of the problem. In this paper we consider the Cauchy problem $(P)$

$$
\begin{cases}u_{t}+[\varphi(u)]_{x}=0 & \text { in } \mathbb{R} \times(0, T)=: S \\ u=u_{0} & \text { in } \mathbb{R} \times\{0\}\end{cases}
$$

Here $T>0, u_{0}$ is a positive Radon measure on $\mathbb{R}$ whose singular part $u_{0 s}$ (with respect to the Lebesgue measure) is a finite superposition of Dirac masses, and $\varphi$ is a smooth and bounded function with bounded derivative:

$$
\begin{gathered}
u_{0 s}=\sum_{j=1}^{p} c_{j} \delta_{x_{j}} \quad\left(x_{1}<x_{2}<\cdots<x_{p} ; \quad c_{j}>0 \text { for } 1 \leq j \leq p\right), \\
\varphi \in C^{2}([0, \infty)) \cap W^{1, \infty}(0, \infty) .
\end{gathered}
$$
It is worth mentioning that problem $(P)$ is related to a class of interesting ap-
plicative models. A common technique for the fabrication of semiconductor devices is the so-called ion etching, in which the material to be etched is bombarded with an ion beam (see 17, 11, 12, ). Mathematical modelling of the process gives rise to the Hamilton-Jacobi equation in one space dimension:

$$
\begin{cases}U_{t}+\varphi\left(U_{x}\right)=0 & \text { in } \mathbb{R} \times(0, T) \\ U=U_{0} & \text { in } \mathbb{R} \times\{0\}\end{cases}
$$

where $U=U(x, t)$ denotes the thickness of the material and $\varphi$ is bounded, nonconvex and vanishing at infinity. Clearly, problem $(H J)$ is related to $(P)$ by formally differentiating with respect to $x$ and setting $u=U_{x}, u_{0}=U_{0}^{\prime}$. In this way space discontinuous solutions of $(H J)$ correspond to Radon measure-valued solutions of

Date: March 28, 2018.

1991 Mathematics Subject Classification. Primary: 35D99, 35K55, 35R25; Secondary: 28A33, $28 \mathrm{~A} 50$.

Key words and phrases. First order hyperbolic conservation laws, Radon measure-valued solutions, entropy inequalities, uniqueness. 
$(P)$, which have a Dirac mass $\delta_{x_{0}}$ concentrated at any point $x_{0}$ where $U(\cdot, t)$ is discontinuous $(t \in(0, T))$.

Problem $(P)$ was studied in [3] under more general hypotheses on $\varphi$ :

$\left(A_{1}\right) \quad \varphi \in C([0, \infty)), \varphi(0)=0, \varphi^{\prime} \in L^{\infty}(0, \infty)$, there exists $\lim _{u \rightarrow \infty} \frac{\varphi(u)}{u}=: C_{\varphi}$.

Without loss of generality one may assume that $C_{\varphi}=0$ (otherwise replace $x$ by $x-C_{\varphi} t$, see [3]). If $u_{0}$ is any positive bounded Radon measure, an approximation approach can be used to construct suitably defined entropy solutions of $(P)$ in a space of bounded Radon measures on $S$ (see Definitions $3.1+3.2$ below and 3 , Theorem 3.2]; in the present section we call such solutions "constructed solutions"). However, an additional condition on solutions is needed for the well-posedness of $(P)$, since examples of nonuniqueness can be easily produced (see 3 , 5]). If $\left(H_{0}\right)$ and $\left(A_{1}\right)$ hold and $\varphi$ is bounded and monotonic, a uniqueness condition is known. It prescribes the behaviour of the regular part $u_{r}$ of the solution at points of the support of its singular part $u_{s}$ :

$$
\left(x_{j}, t\right) \in \operatorname{supp} u_{s} \Rightarrow \begin{cases}\operatorname{ess} \lim _{x \rightarrow x_{j}^{+}} u_{r}(x, t)=\infty & \text { if } \varphi^{\prime}>0 \text { in }[0, \infty) \\ \operatorname{ess} \lim _{x \rightarrow x_{j}^{-}} u_{r}(x, t)=\infty & \text { if } \varphi^{\prime}<0 \text { in }[0, \infty) .\end{cases}
$$

More precisely, in this case there exists at most one entropy solution of $(P)$, which satisfies (1.1) and is strongly continuous at $t=0$ ([3, Theorem 3.11]). If in addition $\left(A_{2}\right) \varphi \in C^{3}([0, \infty)) ; \exists L \geq-1, K \in \mathbb{R}$ such that $\varphi^{\prime \prime}(u)[L \varphi(u)+K] \leq-\left[\varphi^{\prime}(u)\right]^{2}<0$, every constructed entropy solution $u$ of problem $(P)$ satisfies (1.1), thus providing an existence and uniqueness theorem for $(P)$ ([3] Theorem 3.12]).

Observe that $\left(A_{2}\right)$ entails hat $\varphi$ is either increasing and concave or decreasing and convex. It is the aim of this paper to extend the above well-posedness results to the general case of a bounded flux $\varphi$, without assumptions about its monotonicity or convexity. To this purpose, we must find a general condition which replaces (1.1).

1.2. A modified Cauchy problem. Condition (1.1) was suggested by the model problem (see [3])

$$
\left\{\begin{array}{lll}
u_{t}+[\varphi(u)]_{x}=0 & \text { in } S \\
u=\delta_{0} & \text { in } \mathbb{R} \times\{0\}, & \text { with } \varphi(u)=1-(1+u)^{-p} \quad(p>0)
\end{array}\right.
$$

where $T>1$. In fact, the unique constructed entropy solution of problem (1.2) is

(1.3) $u_{r}(x, t):=\left[\left(p t x^{-1}\right)^{\frac{1}{1+p}}-1\right] \chi_{A}(x, t), \quad u_{s}(t):=\max \{1-t, 0\} \delta_{0} \quad((x, t) \in S)$,

where we have set

$$
A:=\{(x, t) \in S \mid 0<x \leq p t, 0 \leq t<1\} \cup\{(x, t) \in S \mid \xi(t) \leq x \leq p t, 1 \leq t \leq T\},
$$

and $\xi(t)$ is defined by

$$
\xi^{\prime}=\frac{1-\left(p t \xi^{-1}\right)^{-\frac{p}{1+p}}}{\left(p t \xi^{-1}\right)^{\frac{1}{1+p}}-1} \quad \text { in }(1, T), \quad \xi(1)=0 .
$$

Observe that the function $u_{r}$ defined in (1.3) diverges as $x \rightarrow 0^{+}$if $t \in(0,1)$ - namely, as long as $u_{s}(\cdot, t)(\{0\})>0$, in agreement with the first equality in (1.1). On the other hand, $u_{r} \equiv 0$ in the halfstrip $S_{-}:=(-\infty, 0) \times(0, T)$, in particular $u_{r}(x, t) \rightarrow 0$ as $x \rightarrow 0^{-}$. 
To generalize (1.1) it is natural to address the "modified Riemann problem":

rimo

$$
\begin{cases}u_{t}+[\varphi(u)]_{x}=0 & \text { in } S \\ u=u_{0} & \text { in } \mathbb{R} \times\{0\}\end{cases}
$$

with

rimod (1.5)

$$
u_{0 r}=u_{-} \chi_{(-\infty, 0)}+u_{+} \chi_{[0, \infty)}, \quad u_{0 s}=\delta_{0} \quad\left(u_{ \pm} \in[0, \infty)\right) .
$$

We seek entropy solutions of (1.4)-(1.5). We introduce the sets $V_{ \pm} \subseteq[0, \infty]$ of points which are visible from the right,

$\mathrm{deV}$

$$
\begin{aligned}
& V_{+}(\varphi):=\{u \in[0, \infty] \mid \varphi(u) \geq \varphi(s) \quad \forall s \in(u, \infty]\} \\
& V_{-}(\varphi):=\{u \in[0, \infty] \mid \varphi(u) \leq \varphi(s) \quad \forall s \in(u, \infty]\},
\end{aligned}
$$

and set

$$
s_{ \pm}\left(\varphi, u_{0}\right):=\inf V_{ \pm}(\varphi) \cap\left[u_{0}, \infty\right] \quad \text { for } u_{0} \in[0, \infty) .
$$

For shortness we often write $V_{ \pm}$instead of $V_{ \pm}(\varphi)$, and $s_{ \pm}$or $s_{ \pm}\left(u_{0}\right)$ instead of $s_{ \pm}\left(\varphi, u_{0}\right)$. Observe that for all $u_{0} \in[0, \infty)$

$s_{+}=\infty, s_{-}=u_{0}$ if $\varphi$ is increasing, $\quad s_{+}=u_{0}, s_{-}=\infty$ if $\varphi$ is decreasing.

The following properties of $s_{ \pm}$are easily checked:

$$
\begin{gathered}
s_{+}=\inf \left\{u \in\left[u_{0}, \infty\right] \mid \sup _{\left[u_{0}, u\right)} \varphi=\sup _{\left[u_{0}, \infty\right)} \varphi\right\}, \\
s_{-}=\inf \left\{u \in\left[u_{0}, \infty\right] \mid \inf _{\left[u_{0}, u\right)} \varphi=\inf _{\left[u_{0}, \infty\right)} \varphi\right\} ; \\
s_{ \pm} \in V_{ \pm} \text {, hence } s_{ \pm}\left(\varphi, u_{0}\right)=\min V_{ \pm}(\varphi) \cap\left[u_{0}, \infty\right] .
\end{gathered}
$$

Plainly, it follows from (1.7)-(1.8) that

SS

mima

mimabis

p0-

rv+

rv-

$$
\begin{gathered}
s_{ \pm}\left(s_{ \pm}\left(u_{0}\right)\right)=s_{ \pm}\left(u_{0}\right), \\
\varphi\left(s_{+}\left(u_{0}\right)\right)= \begin{cases}\operatorname{lup}_{\left[s_{+}\left(u_{0}\right), \infty\right)} \varphi & \text { if } s_{+}\left(u_{0}\right)<\infty \\
\limsup _{s \rightarrow \infty} \varphi(s) & \text { if } s_{+}\left(u_{0}\right)=\infty,\end{cases} \\
\varphi\left(s_{-}\left(u_{0}\right)\right)= \begin{cases}\inf _{\left[s_{-}\left(u_{0}\right), \infty\right) \varphi} & \text { if } s_{-}\left(u_{0}\right)<\infty \\
\liminf _{s \rightarrow \infty} \varphi(s) & \text { if } s_{-}\left(u_{0}\right)=\infty,\end{cases}
\end{gathered}
$$

whence

$$
\begin{gathered}
\varphi^{\prime}\left(s_{ \pm}\left(u_{0}\right)\right)=0 \quad \text { if } u_{0}<s_{ \pm}\left(u_{0}\right)<\infty, \\
\varphi^{\prime}\left(s_{+}\left(u_{0}\right)\right) \leq 0 \quad \text { if } s_{+}\left(u_{0}\right)=u_{0}, \quad \varphi^{\prime}\left(s_{-}\left(u_{0}\right)\right) \geq 0 \quad \text { if } s_{-}\left(u_{0}\right)=u_{0},
\end{gathered}
$$

and

$$
\varphi\left(s_{-}\left(u_{0}\right)\right) \leq \liminf _{s \rightarrow \infty} \varphi(s) \leq \limsup _{s \rightarrow \infty} \varphi(s) \leq \varphi\left(s_{+}\left(u_{0}\right)\right) \quad \text { for } u_{0} \in[0, \infty] .
$$

Now consider the Riemann problems:

$$
\begin{cases}v_{t}+[\varphi(v)]_{x}=0 & \text { in } S \\ v=s_{+}\left(u_{+}\right) \chi_{(-\infty, 0)}+u_{+} \chi_{[0, \infty)} & \text { in } \mathbb{R} \times\{0\},\end{cases}
$$

$$
\begin{cases}v_{t}+[\varphi(v)]_{x}=0 & \text { in } S \\ v=u_{-} \chi_{(-\infty, 0)}+s_{-}\left(u_{-}\right) \chi_{[0, \infty)} & \text { in } \mathbb{R} \times\{0\}\end{cases}
$$


with $s_{ \pm}\left(u_{ \pm}\right)<\infty$. Denote by $v_{ \pm}$the unique entropy solution of (1.14) and (1.15), respectively. If $u_{ \pm}=s_{ \pm}\left(u_{ \pm}\right)$, there holds $v_{ \pm} \equiv s_{ \pm}\left(u_{ \pm}\right)$in $S$. On the other hand, if $u_{ \pm}<s_{ \pm}\left(u_{ \pm}\right), v_{ \pm}$can be constructed in a standard way by considering the convex hull of $\varphi$ in the interval $\left[u_{-}, s_{-}\left(u_{-}\right)\right]$, respectively its concave hull in the interval $\left[u_{+}, s_{+}\left(u_{+}\right)\right]$, and the corresponding characteristics. Plainly, by (1.12) there holds

$$
v_{ \pm}=s_{ \pm}\left(u_{ \pm}\right) \quad \text { in } S_{\mp},
$$

where $S_{+}:=(0, \infty) \times(0, T)$ and $S_{-}:=(-\infty, 0) \times(0, T) ;$ moreover,

trari

$$
\exists \lim _{x \rightarrow 0^{ \pm}} v_{ \pm}(x, t)=: v_{ \pm}\left(0^{ \pm}, t\right), \quad \text { and } \quad v_{ \pm}\left(0^{ \pm}, t\right)=s_{ \pm}\left(u_{ \pm}\right) \text {for all } t \in(0, T) .
$$

Now we can construct an entropy solution of problem (1.4). Set

defini

$$
u_{r}(\cdot, t):=\left\{\begin{array}{ll}
v_{-}(\cdot, t) & \text { in }(-\infty, 0) \\
v_{+}(\cdot, t) & \text { in }(0, \infty)
\end{array}, \quad u_{s}(\cdot, t):=A(t) \delta_{0}\right.
$$

where

$$
A(t):=1-\left[\varphi\left(s_{+}\left(u_{+}\right)\right)-\varphi\left(s_{-}\left(u_{-}\right)\right)\right] t .
$$

Observe that $\varphi\left(s_{+}\left(u_{+}\right)\right) \geq \varphi\left(s_{-}\left(u_{-}\right)\right)$by (1.13). If $\varphi\left(s_{+}\left(u_{+}\right)\right)>\varphi\left(s_{-}\left(u_{-}\right)\right)$, the measure $u$ defined by (1.18)-(1.19) is positive on $\mathbb{R} \times(0, \tau)$ with

$$
\tau:=\frac{1}{\varphi\left(s_{+}\left(u_{+}\right)\right)-\varphi\left(s_{-}\left(u_{-}\right)\right)} .
$$

It is easily seen that $u$ is an entropy solution of the modified Riemann problem (1.4) in $\mathbb{R} \times(0, \min \{\tau, T\})$ (see Definitions 3.1 3.2). By (1.17)-(1.18), there holds

$$
u_{r}\left(0^{ \pm}, t\right):=\lim _{x \rightarrow 0^{ \pm}} u_{r}(x, t)=s_{ \pm}\left(u_{ \pm}\right) \quad \text { for all } t \in(0, \min \{\tau, T\}) .
$$

If $\tau \geq T$, the result follows. Otherwise, we set $u_{s}(\cdot, t):=0$ for all $t \in(\tau, T]$ and continue the solution in $(\tau, T]$, with initial data $u_{r}(\cdot, \tau)$, using the standard theory of scalar conservation laws. If $\varphi\left(s_{+}\left(u_{+}\right)\right)=\varphi\left(s_{-}\left(u_{-}\right)\right)$, it is easily seen that $u$ is an equilibrium solution in $S: A(t) \equiv 1$ in [0,T], and, by (1.13) and the definition of $s_{ \pm}$, $\varphi \equiv \varphi\left(s_{+}\left(u_{+}\right)=\varphi\left(s_{-}\left(u_{-}\right)\right.\right.$in the interval $\left(\min \left\{u_{-}, u_{+}\right\}, \infty\right)$, thus $u_{r}$ is constant in $S$ (see (1.18) ). One easily generalizes the above discussion to the case that $s_{ \pm}\left(u_{ \pm}\right)=\infty$.

It is worth revisiting problem (1.2) in the light of the above remarks. Since in this case $u_{ \pm}=0$ and $\varphi$ is increasing, there holds $s_{+}\left(u_{+}\right)=\infty, s_{-}\left(u_{-}\right)=0, \varphi\left(s_{+}\left(u_{+}\right)\right)=1$ and $\varphi\left(s_{-}\left(u_{-}\right)\right)=0$, whence (see (1.20) $)$

$$
\lim _{x \rightarrow 0^{+}} u_{r}(x, t)=\infty, \quad \lim _{x \rightarrow 0^{-}} u_{r}(x, t)=0, \quad A(t)=1-t \quad \text { for } t \in[0,1],
$$

in agreement with (1.3).

1.3. Compatibility conditions. To address problem $(P)$ under assumption $\left(H_{0}\right)$ we need a more general condition than (1.20), which is only suitable for the modified Riemann problem. To this purpose, observe that equalities (1.9) and (1.20) entail

$$
u_{r}\left(0^{ \pm}, t\right)=s_{ \pm}\left(u_{r}\left(0^{ \pm}, t\right)\right)
$$

for all $t \in(0, T)$ such that $u_{s}(\cdot, t)>0$. For problem (1.2) the equality at $0^{+}$coincides with the first equality in (1.1), while that at $0^{-}$is trivially satisfied. So, if $\{0\} \times(0, t) \in$ $\operatorname{supp} u_{s}$, it is natural to regard (1.21) as the desired generalization of (1.1). 
Set $H_{-}(u):=-\chi_{(-\infty, 0)}(u)(u \in \mathbb{R})$. It is easily seen that condition (1.21) can be rephrased as

$$
\begin{aligned}
& H_{-}\left(u_{r}\left(0^{+}, t\right)-k\right)\left[\varphi\left(u_{r}\left(0^{+}, t\right)\right)-\varphi(k)\right] \leq 0 \\
& H_{-}\left(u_{r}\left(0^{-}, t\right)-k\right)\left[\varphi\left(u_{r}\left(0^{-}, t\right)\right)-\varphi(k)\right] \geq 0
\end{aligned} \quad \text { for all } k \in[0, \infty) .
$$

Formally (1.22) is equivalent to the compatibility condition

trariqui

$$
\begin{aligned}
& {\left[\operatorname{sgn}\left(u_{r}\left(0^{+}, t\right)-k\right)-\operatorname{sgn}\left(a_{0}(t)-k\right)\right]\left[\varphi\left(u_{r}\left(0^{+}, t\right)\right)-\varphi(k)\right] \leq 0} \\
& {\left[\operatorname{sgn}\left(u_{r}\left(0^{-}, t\right)-k\right)-\operatorname{sgn}\left(a_{0}(t)-k\right)\right]\left[\varphi\left(u_{r}\left(0^{-}, t\right)\right)-\varphi(k)\right] \geq 0}
\end{aligned}
$$

between the traces $u_{r}\left(0^{ \pm}, t\right)$ and the boundary data $a_{0}(t)=\infty$, for all $k, t$ as above. It was shown in 2, 14, that the initial-boundary value problems

$$
\left\{\begin{array} { l l } 
{ v _ { t } + [ \varphi ( v ) ] _ { x } = 0 } & { \text { in } S _ { + } } \\
{ v = a _ { 0 } } & { \text { in } \{ 0 \} \times ( 0 , T ) } \\
{ v = u _ { + } } & { \text { in } [ 0 , \infty ) \times \{ 0 \} }
\end{array} \quad \left\{\begin{array}{ll}
v_{t}+[\varphi(v)]_{x}=0 & \text { in } S_{-} \\
v=a_{0} & \text { in }\{0\} \times(0, T) \\
v=u_{-} & \text {in }(-\infty, 0] \times\{0\}
\end{array}\right.\right.
$$

are well posed, if $a_{0} \in B V(0, T)$ and (1.23) holds. This gives an alternative interpretation of the construction used to solve the modified Riemann problem (1.4) (see (1.14)-(1.15)): as long as the Dirac delta at $x=0$ survives, it behaves like a barrier which decouples the evolution of the regular part of the solution on either side of the singularity, imposing the two Dirichlet conditions $u_{r}\left(0^{ \pm}, t\right)=\infty$ at $x=0$. The evolution of the delta at $t=0$ is completely determined by local mass exchange through $x=0$.

The above considerations suggest a constructive approach to address problem $(P)$ under assumption $\left(H_{0}\right)$. By the results in [3] there is a positive time $\tau$ until which all singularities persist, thus the real line is the disjoint union of $p+1$ intervals. In each interval we solve the initial-boundary value problem for the conservation law in $(P)$, the initial data being the restriction of $u_{0 r}$ to that interval, with "boundary conditions equal to infinity" - or, equivalently, by imposing the analogue of (1.22) to be satisfied at each point $x_{j}, j=1, \ldots, p$. The function determined by this procedure is, by definition, the regular part of a Radon measure, whose singular part is defined in analogy with (1.18)-(1.19). It is proven that this measure is the unique entropy solution of $(P)$ (in the sense of Definitions 3.1]3.2) until the time $t=\tau$. If $\tau<T$ we iterate the procedure in $\mathbb{R} \times(\tau, T)$ with a smaller number a singularities, thus well-posedness of $(P)$ follows in a finite number of steps (see Theorem 3.1).

A technical obstruction to the above program is that the solution constructed in each interval need not have traces at the points $x_{j}$. This difficulty is overcome by using a weak analogue of condition (1.22) (see (3.7)) and the $L^{\infty}$-theory of initialboundary value problems developed in [10].

By the finite speed of propagation of solutions of hyperbolic conservation laws, uniqueness proofs are local in space. Then it can be easily checked that our results remain valid, if condition $\left(H_{0}\right)$ is relaxed to the case that $u_{0 s}$ is a locally finite superposition of Dirac masses (namely, in every bounded interval the number of Dirac masses is finite). The case of more general $u_{0 s}$ is open.

The paper is organized as follows. After recalling some preliminaries (see Section 2), the main results of the paper are presented in Section 3, whereas Sections 4,6 are devoted to their proofs. 


\section{Preliminaries}

Let $\chi_{E}$ denote the characteristic function of $E \subseteq \mathbb{R}$. For all $u \in \mathbb{R}$, we set $u_{ \pm}=\max \{ \pm u, 0\}, H_{ \pm}(u)= \pm \chi_{\{ \pm u>0\}}(u), \operatorname{sgn}(u)=H_{+}(u)+H_{-}(u)$. For every real function $f$ on $\mathbb{R}$ and $x_{0} \in \mathbb{R}$ we say that

$$
\text { ess } \lim _{x \rightarrow x_{0}^{ \pm}} f(x)=l \in \mathbb{R},
$$

if there is a null set $E^{*} \subseteq \mathbb{R}$ such that $f\left(x_{n}\right) \rightarrow l$ if $\left\{x_{n}\right\} \subseteq \mathbb{R} \backslash\left(E^{*} \cup\left\{x_{0}\right\}\right), x_{n} \rightarrow x_{0}^{ \pm}$.

For every open subset $\Omega \subseteq \mathbb{R}$ we denote by $\mathcal{M}(\Omega)$ the space of Radon measures on $\Omega$, by $\mathcal{M}^{+}(\Omega)$ the cone of its nonnegative elements. If $\mu, \nu \in \mathcal{M}(\Omega)$, we say that $\mu \leq \nu$ in $\mathcal{M}(\Omega)$ if $\nu-\mu \in \mathcal{M}^{+}(\Omega)$. We denote by $C_{c}(\Omega)$ the space of continuous real functions with compact support in $\Omega$, and by $\langle\cdot, \cdot\rangle_{\Omega}$ the duality map between $\mathcal{M}(\Omega)$ and $C_{c}(\Omega)$. A sequence $\left\{\mu_{n}\right\}$ of Radon measures on $\mathbb{R}$ converges weakly* to a Radon measure $\mu, \mu_{n} \stackrel{*}{\rightarrow} \mu$, if $\left\langle\mu_{n}, \rho\right\rangle_{\mathbb{R}} \rightarrow\langle\mu, \rho\rangle_{\mathbb{R}}$ for all $\rho \in C_{c}(\mathbb{R})$. For any compact $K \in \mathbb{R}$ the space $\mathcal{M}(K)$ is a Banach space with norm $\|\mu\|_{\mathcal{M}(K)}:=|\mu|(K)$. A sequence $\left\{\mu_{n}\right\}$ converges strongly to $\mu$ in $\mathcal{M}(K)$ if $\left\|\mu_{n}-\mu\right\|_{\mathcal{M}(K)} \rightarrow 0$ as $n \rightarrow \infty$. Similar definitions are used for Radon measures on any subset of $S:=\mathbb{R} \times(0, T)$.

Every $\mu \in \mathcal{M}(\mathbb{R})$ has a unique decomposition $\mu=\mu_{a c}+\mu_{s}$, with $\mu_{a c} \in \mathcal{M}(\mathbb{R})$ absolutely continuous and $\mu_{s} \in \mathcal{M}(\mathbb{R})$ singular with respect to the Lebesgue measure. We denote by $\mu_{r} \in L_{\text {loc }}^{1}(\mathbb{R})$ the density of $\mu_{a c}$. Every function $f \in L_{\text {loc }}^{1}(\mathbb{R})$ can be identified to an absolutely continuous Radon measure on $\mathbb{R}$; we shall denote this measure by the same symbol $f$ used for the function.

The restriction $\mu\llcorner E$ of $\mu \in \mathcal{M}(\mathbb{R})$ to a Borel set $E \subseteq \mathbb{R}$ is defined by $(\mu\llcorner E)(A):=$ $\mu(E \cap A)$ for any Borel set $A \subseteq \mathbb{R}$. Similar notations are used for $\mathcal{M}(S)$.

We shall use measures $u \in \mathcal{M}(S)$ which, roughly speaking, admit a parametrization with respect to the time variable:

dli Definition 2.1. We denote by $L^{\infty}\left(0, T ; \mathcal{M}^{+}(\mathbb{R})\right)$ the set of nonnegative Radon measures $u \in \mathcal{M}^{+}(S)$ such that for a.e. $t \in(0, T)$ there is a measure $u(\cdot, t) \in \mathcal{M}^{+}(\mathbb{R})$ with the following properties:

$(i)$ if $\zeta \in C\left([0, T] ; C_{c}(\mathbb{R})\right)$ the map $t \mapsto\langle u(\cdot, t), \zeta(\cdot, t)\rangle_{\mathbb{R}}$ belongs to $L^{1}(0, T)$ and

$$
\langle u, \zeta\rangle_{S}=\int_{0}^{T}\langle u(\cdot, t), \zeta(\cdot, t)\rangle_{\mathbb{R}} d t ;
$$

(ii) the map $t \mapsto\|u(\cdot, t)\|_{\mathcal{M}(K)}$ belongs to $L^{\infty}(0, T)$ for every compact $K \in \mathbb{R}$.

Remark 2.1. Definition2.1 implies that for all $\rho \in C_{c}(\mathbb{R})$ the map $t \mapsto\langle u(\cdot, t), \rho\rangle_{\mathbb{R}}$ is measurable, thus the map $u:(0, T) \rightarrow \mathcal{M}(\mathbb{R})$ is weakly* measurable. For simplicity we prefer the notation $L^{\infty}(0, T ; \mathcal{M}(\mathbb{R}))$ to the more correct one $L_{w *}^{\infty}(0, T ; \mathcal{M}(\mathbb{R}))$.

Observe that $u_{r} \in L^{\infty}\left(0, T ; L_{l o c}^{1}(\mathbb{R})\right)$ if $u \in L^{\infty}\left(0, T ; \mathcal{M}^{+}(\mathbb{R})\right)$. Conversely, every nonnegative $f \in L^{\infty}\left(0, T ; L_{\text {loc }}^{1}(\mathbb{R})\right)$ defines a measure belonging to $L^{\infty}\left(0, T ; \mathcal{M}^{+}(\mathbb{R})\right)$.

By $C\left([0, T] ; \mathcal{M}^{+}(\mathbb{R})\right)$ we denote the subset of strongly continuous mappings from $[0, T]$ into $\mathcal{M}^{+}(\mathbb{R})$ - namely, $u \in C\left([0, T] ; \mathcal{M}^{+}(\mathbb{R})\right)$ if for all $t_{0} \in[0, T]$ and for every compact $K \in \mathbb{R}$ there holds $\left\|u(\cdot, t)-u\left(\cdot, t_{0}\right)\right\|_{\mathcal{M}(K)} \rightarrow 0$ as $t \rightarrow t_{0}$.

If $u \in L^{\infty}\left(0, T ; \mathcal{M}^{+}(\mathbb{R})\right)$, also $u_{a c}, u_{s} \in L^{\infty}\left(0, T ; \mathcal{M}^{+}(\mathbb{R})\right)$ and, by (2.1),

disicomp

$$
\left\langle u_{a c}, \zeta\right\rangle_{S}=\iint_{S} u_{r} \zeta d x d t, \quad\left\langle u_{s}, \zeta\right\rangle_{S}=\int_{0}^{T}\left\langle u_{s}(\cdot, t), \zeta(\cdot, t)\right\rangle_{\mathbb{R}} d t
$$

if $\zeta \in C\left([0, T] ; C_{c}(\mathbb{R})\right)$. One easily checks that for a.e. $t \in(0, T)$

$u s(t)=u(t) s$

$$
u_{a c}(\cdot, t)=[u(\cdot, t)]_{a c}, \quad u_{s}(\cdot, t)=[u(\cdot, t)]_{s}, \quad u_{r}(\cdot, t)=[u(\cdot, t)]_{r},
$$


where $[u(\cdot, t)]_{r}$ denotes the density of the measure $[u(\cdot, t)]_{a c}$ : for $\rho \in C_{c}(\mathbb{R})$

$$
\left\langle[u(\cdot, t)]_{a c}, \rho\right\rangle_{\mathbb{R}}=\int_{\mathbb{R}}[u(\cdot, t)]_{r} \rho d x=\int_{\mathbb{R}} u_{r}(\cdot, t) \rho d x \quad \text { for a.e. } t \in(0, T) .
$$

In view of (2.2)-(2.3), we shall always identify the quantities which appear on either side of equalities (2.3).

\section{REsults}

For any open $\Omega \subseteq \mathbb{R}$ and $\tau \in(0, T)$ set $Q_{\tau}:=\Omega \times(0, \tau)$. Solutions of problem $(P)$ are meant in the following sense.

deso Definition 3.1. A measure $u \in L^{\infty}\left(0, T ; \mathcal{M}^{+}(\Omega)\right)$ is called a solution of problem $(P)$ in $Q_{\tau}$ if for all $\zeta \in C^{1}\left([0, \tau] ; C_{c}^{1}(\Omega)\right), \zeta(\cdot, \tau)=0$ in $\Omega$ there holds

$$
\iint_{Q_{\tau}}\left[u_{r} \zeta_{t}+\varphi\left(u_{r}\right) \zeta_{x}\right] d x d t+\int_{0}^{\tau}\left\langle u_{s}(\cdot, t), \zeta_{t}(\cdot, t)\right\rangle_{\Omega} d t=-\left\langle u_{0}, \zeta(\cdot, 0)\right\rangle_{\Omega} .
$$

Solutions of $(P)$ in $S$ are simply referred to as "solutions of $(P)$ ".

enso Definition 3.2. A solution of $(P)$ in $Q_{\tau}$ is called an entropy solution in $Q_{\tau}$ if it satisfies the entropy inequality

$$
\begin{aligned}
& \iint_{Q_{\tau}}\left\{\left|u_{r}-k\right| \zeta_{t}+\operatorname{sgn}\left(u_{r}-k\right)\left[\varphi\left(u_{r}\right)-\varphi(k)\right] \zeta_{x}\right\} d x d t+ \\
+ & \int_{0}^{\tau}\left\langle u_{s}(\cdot, t), \zeta_{t}(\cdot, t)\right\rangle_{\Omega} d t \geq-\int_{\Omega}\left|u_{0 r}(x)-k\right| \zeta(x, 0) d x-\left\langle u_{0 s}, \zeta(\cdot, 0)\right\rangle_{\Omega}
\end{aligned}
$$

for all $\zeta \in C^{1}\left([0, \tau] ; C_{c}^{1}(\Omega)\right), \zeta \geq 0, \zeta(\cdot, \tau)=0$ in $\Omega$, and for all $k \in[0, \infty)$.

susu Remark 3.1. Entropy subsolutions and supersolutions of $(P)$ in $Q_{\tau}$ are defined by requiring the following inequalities to be satisfied:

$$
\begin{gathered}
\iint_{Q_{\tau}}\left\{\left[u_{r}-k\right]_{+} \zeta_{t}+H_{+}\left(u_{r}-k\right)\left[\varphi\left(u_{r}\right)-\varphi(k)\right] \zeta_{x}\right\} d x d t+ \\
+\quad \int_{0}^{\tau}\left\langle u_{s}(\cdot, t), \zeta_{t}(\cdot, t)\right\rangle_{\Omega} d t \geq-\int_{\Omega}\left[u_{0 r}-k\right]_{+} \zeta(x, 0) d x-\left\langle u_{0 s}, \zeta(\cdot, 0)\right\rangle_{\Omega},
\end{gathered}
$$

respectively

superkru

$$
\iint_{Q_{\tau}}\left\{\left[u_{r}-k\right]_{-} \zeta_{t}+H_{-}\left(u_{r}-k\right)\left[\varphi\left(u_{r}\right)-\varphi(k)\right] \zeta_{x}\right\} d x d t \geq-\int_{\Omega}\left[u_{0 r}-k\right]_{-} \zeta(x, 0) d x
$$

for all $\zeta$ and $k$ as above. It is easily seen that $u$ is an entropy solution if and only if it is both an entropy subsolution and an entropy supersolution.

Let $\beta \in C_{c}^{1}(0, T), \beta \geq 0$, and $k \in[0, \infty)$. We shall prove below (see Lemma 4.2) that, if $\left(H_{0}\right)-\left(H_{1}\right)$ hold, for every entropy solution of $(P)$ the limits

$$
\text { ess } \lim _{x \rightarrow x_{j}^{ \pm}} \int_{0}^{T} H_{-}\left(u_{r}(x, t)-k\right)\left[\varphi\left(u_{r}(x, t)\right)-\varphi(k)\right] \beta(t) d t \quad(j=1, \ldots, p)
$$

exist and are finite. It is also known that, if $\left(H_{0}\right)-\left(H_{1}\right)$ are satisfied, $j=1, \ldots, p$ and $u$ is a solution of problem $(P)$,

$$
\forall x_{j} \exists t_{j} \in(0, T] \text { such that } \begin{cases}u_{s}(\cdot, t)\left(\left\{x_{j}\right\}\right)>0 & \text { for a.e. } t \in\left[0, t_{j}\right) \\ u_{s}(\cdot, t)\left(\left\{x_{j}\right\}\right)=0 & \text { for a.e. } t \in\left(t_{j}, T\right)\end{cases}
$$

(see [3, Theorem 3.5]). Then we can state the following definition. 
decoco Definition 3.3. Let $\left(H_{0}\right)-\left(H_{1}\right)$ be satisfied, let $j=1, \ldots, p$ and let $\tau \in\left(0, t_{j}\right]$. An entropy solution of $(P)$ satisfies the compatibility condition at $x_{j}$ in $[0, \tau]$ if for all $\beta \in C_{c}^{1}(0, \tau), \beta \geq 0$, and $k \in[0, \infty)$

$$
\begin{aligned}
& \text { ess } \lim _{x \rightarrow x_{j}^{+}} \int_{0}^{\tau} H_{-}\left(u_{r}(x, t)-k\right)\left[\varphi\left(u_{r}(x, t)\right)-\varphi(k)\right] \beta(t) d t \leq 0, \\
& \text { ess } \lim _{x \rightarrow x_{j}^{-}} \int_{0}^{\tau} H_{-}\left(u_{r}(x, t)-k\right)\left[\varphi\left(u_{r}(x, t)\right)-\varphi(k)\right] \beta(t) d t \geq 0 .
\end{aligned}
$$

Now our main result can be stated as follows.

exiuni Theorem 3.1. Let $\left(H_{0}\right)-\left(H_{1}\right)$ be satisfied. Then there exists a unique entropy solution of problem $(P)$ which belongs to $C\left([0, T] ; \mathcal{M}^{+}(\mathbb{R})\right)$ and satisfies the compatibility condition at $x_{j}$ in $\left[0, t_{j}\right]$ for all $j=1, \ldots, p$.

According to Theorem 3.1, the compatibility condition defines a well-posedness class for entropy solutions of $(P)$ under assumptions $\left(H_{0}\right)-\left(H_{1}\right)$.

We shall also prove a comparison result for solutions of $(P)$ whose initial data satisfy assumption $\left(H_{0}\right)$ :

compa Theorem 3.2. Let $\left(H_{1}\right)$ be satisfied. Let $v_{0} \in \mathcal{M}^{+}(\mathbb{R})$ satisfy $\left(H_{0}\right)$, and let $u_{0} \leq v_{0}$ in $\mathcal{M}(\mathbb{R})$. Let $u, v \in C\left([0, T] ; \mathcal{M}^{+}(\mathbb{R})\right)$ be the unique entropy solutions of $(P)$ with initial data $u_{0}, v_{0}$ given by Theorem [3.1. Then there holds $u(\cdot, t) \leq v(\cdot, t)$ in $\mathcal{M}(\mathbb{R})$ for all $t \in[0, T]$.

prexi

\section{Proof of existence}

In this section we prove the existence part of Theorem 3.1

exi Theorem 4.1. Let $\left(H_{0}\right)-\left(H_{1}\right)$ be satisfied. Then there exists an entropy solution of problem $(P)$ which satisfies the compatibility condition at $x_{j}$ in $\left[0, t_{j}\right]$ for all $j=1, \ldots, p$. Moreover, $u$ belongs to $C\left([0, T] ; \mathcal{M}^{+}(\mathbb{R})\right)$.

To prove Theorem 4.1 we need some preliminary results.

\subsection{Preliminary results.}

$\lim$ Lemma 4.2. Let $u$ be an entropy supersolution of $(P)$, and let $\beta \in C_{c}^{1}(0, T), \beta \geq 0$. Then:

(i) for every $k \in[0, \infty)$ the distributional derivative of the function

$$
x \mapsto-\int_{0}^{T} H_{-}\left(u_{r}(x, t)-k\right)\left[\varphi\left(u_{r}(x, t)\right)-\varphi(k)\right] \beta(t) d t+k T\left\|\beta^{\prime}\right\|_{\infty} x
$$

is nonnegative;

(ii) for every $x_{0} \in \mathbb{R}$ and $k \in[0, \infty)$ the limits

ali

$$
\text { ess } \lim _{x \rightarrow x_{0}^{ \pm}} \int_{0}^{T} H_{-}\left(u_{r}(x, t)-k\right)\left[\varphi\left(u_{r}(x, t)\right)-\varphi(k)\right] \beta(t) d t
$$

exist and are finite.

Proof. Let $\alpha \in C_{c}^{1}(\mathbb{R}), \alpha \geq 0$. Choosing $\zeta(x, t)=\alpha(x) \beta(t)$ in 3.4 with $Q_{\tau}=S$ gives $\iint_{S}\left\{\left[u_{r}(x, t)-k\right]_{-} \alpha(x) \beta^{\prime}(t)+H_{-}\left(u_{r}(x, t)-k\right)\left[\varphi\left(u_{r}(x, t)\right)-\varphi(k)\right] \alpha^{\prime}(x) \beta(t)\right\} d x d t \geq 0$.

Since $0 \leq\left[u_{r}-k\right]_{-} \leq k$, from the above inequality we get

$$
-\int_{\mathbb{R}}\left(\int_{0}^{T} H_{-}\left(u_{r}(x, t)-k\right)\left[\varphi\left(u_{r}(x, t)\right)-\varphi(k)\right] \beta(t) d t\right) \alpha^{\prime}(x) d x \leq k T\left\|\beta^{\prime}\right\|_{\infty} \int_{\mathbb{R}} \alpha(x) d x,
$$


whence claim $(i)$ follows.

Therefore, the distributional derivative of function (4.1) is a Radon measure. Clearly, the same holds for the distributional derivative, say $\mu$, of the function $\mathcal{H} \in L_{\text {loc }}^{1}(\mathbb{R})$,

$$
\mathcal{H}(x):=-\int_{0}^{T} H_{-}\left(u_{r}(x, t)-k\right)\left[\varphi\left(u_{r}(x, t)\right)-\varphi(k)\right] \beta(t) d t .
$$

Fix any $\bar{x} \in \mathbb{R}$ and set $f_{\mu}(x):=\mu((\bar{x}, x])$ if $x \geq \bar{x}, f_{\mu}(x):=-\mu((x, \bar{x}])$ if $x<\bar{x}$. Then $f_{\mu}$ is continuous from the right, and coincides a.e. with $\mathcal{H}$ on every compact $K \subset \mathbb{R}$ up to a constant, possibly depending on $K($ e.g., see [1, Theorem 3.28]). Hence the claim follows.

In the following we set $I_{1}:=\left(-\infty, x_{1}\right), I_{j}:=\left(x_{j-1}, x_{j}\right)$ for $j=2, \ldots, p, I_{p+1}:=$ $\left(x_{p}, \infty\right)$ and $S_{j}:=I_{j} \times(0, T)$ for $j=1, \ldots, p+1$.

lime Lemma 4.3. Let $\left(H_{0}\right)-\left(H_{1}\right)$ hold, and let $u$ be an entropy solution of $(P)$. Then for all $\beta \in C_{c}^{1}(0, T), \beta \geq 0, k \in[0, \infty)$ and $j=1, \ldots, p$ the limits

alibis

$$
\text { ess } \lim _{x \rightarrow x_{j}^{ \pm}} \int_{0}^{T} \operatorname{sgn}\left(u_{r}(x, t)-k\right)\left[\varphi\left(u_{r}(x, t)\right)-\varphi(k)\right] \beta(t) d t
$$

exist and are finite.

Proof. We only prove the claim for the limit from the right, the proof being similar for the other. Let $j=1, \ldots, p$ be fixed. Since $\varphi$ is bounded, by 3. Proposition 3.3] the singular part of every entropy solution of $(P)$ is nonincreasing in time, hence $u_{s}(\cdot, t)\left(I_{j+1}\right)=0$ for any $t \in[0, T]$. Let $\alpha \in C_{c}^{1}\left(I_{j+1}\right), \alpha \geq 0$. Choosing $\zeta(x, t)=\alpha(x) \beta(t)$ in 3.2 with $Q_{\tau}=S$ gives

$\iint_{S_{j+1}}\left\{\left|u_{r}(x, t)-k\right| \alpha(x) \beta^{\prime}(t)+\operatorname{sgn}\left(u_{r}(x, t)-k\right)\left[\varphi\left(u_{r}(x, t)\right)-\varphi(k)\right] \alpha^{\prime}(x) \beta(t)\right\} d x d t \geq 0$.

Since $0 \leq\left|u_{r}-k\right| \leq u_{r}+k$, we have

$$
\begin{aligned}
& -\int_{I_{j+1}} d x \alpha^{\prime}(x)\left(\int_{0}^{T} \operatorname{sgn}\left(u_{r}(x, t)-k\right)\left[\varphi\left(u_{r}(x, t)\right)-\varphi(k)\right] \beta(t) d t\right) \leq \\
\leq & \left\|\beta^{\prime}\right\|_{\infty} \int_{I_{j+1}} d x \alpha(x)\left(\int_{0}^{T} u_{r}(x, t) d t+k T\right)= \\
= & -\left\|\beta^{\prime}\right\|_{\infty} \int_{I_{j+1}} d x \alpha^{\prime}(x)\left(\int_{0}^{T} \int_{x_{j}}^{x} u_{r}(y, t) d y d t+k T x\right) .
\end{aligned}
$$

The above inequality implies that the distributional derivative of the map

$$
\begin{aligned}
x \mapsto & -\int_{0}^{T} \operatorname{sgn}\left(u_{r}(x, t)-k\right)\left[\varphi\left(u_{r}(x, t)\right)-\varphi(k)\right] \beta(t) d t+ \\
& +\left\|\beta^{\prime}\right\|_{\infty}\left(\int_{0}^{T} \int_{x_{j}}^{x} u_{r}(y, t) d y d t+k T x\right)
\end{aligned}
$$

is nonnegative in $I_{j+1}$. Arguing as in the proof of Lemma 4.2 the claim follows.

incom Lemma 4.4. Let $\left(H_{0}\right)-\left(H_{1}\right)$ hold, and let $u$ be an entropy solution of $(P)$. Then for every $j=1, \ldots, p$ :

(i) there exist $h_{j}^{-}, h_{j}^{+} \in L^{\infty}(0, T), h_{j}^{ \pm} \geq 0$ such that for all $\beta \in C_{c}^{1}(0, T)$

$$
\operatorname{ess} \lim _{x \rightarrow x_{j}^{ \pm}} \int_{0}^{T} \varphi\left(u_{r}(x, t)\right) \beta(t) d t=\int_{0}^{T} h_{j}^{ \pm}(t) \beta(t) d t ;
$$


(ii) if $u$ satisfies the compatibility condition (3.7) at $x_{j}$ in $[0, \tau]$, there holds

$$
h_{j}^{-} \leq \liminf _{k \rightarrow \infty} \varphi(k) \leq \limsup _{k \rightarrow \infty} \varphi(k) \leq h_{j}^{+} \quad \text { a.e. in }(0, \tau) .
$$

Remark 4.1. By standard density arguments, from (4.4) we get

lidesi1

$$
\text { ess } \lim _{x \rightarrow x_{j}^{ \pm}} \int_{0}^{T} \varphi\left(u_{r}(x, t)\right) \zeta(x, t) d t=\int_{0}^{T} h_{j}^{ \pm}(t) \zeta\left(x_{j}, t\right) d t
$$

for every $\zeta \in L^{1}\left(0, T ; C_{c}\left(U_{j}\right)\right)$ with $x_{j} \in U_{j} \subseteq \mathbb{R}, U_{j}$ open.

Proof of Lemma 4.4. (i) We only prove the limit from the right. Since $\operatorname{sgn} u=$ $1+2 H_{-}(u)$ for $u \in \mathbb{R}$, by (4.2)-(4.3) the limit in the left-hand side of (4.4) exists and is finite. On the other hand, for every sequence $\left\{x_{n}\right\}$ converging to $x_{j}^{+}$the sequence $\left\{\varphi\left(x_{n}\right)\right\}$ is bounded in $L^{\infty}(0, T)$, hence there exist a subsequence $\left\{x_{n_{k}}\right\} \subseteq\left\{x_{n}\right\}$ and a function $h_{j}^{+} \in L^{\infty}(0, T)$ such that $\varphi\left(x_{n_{k}}\right) \stackrel{*}{\rightarrow} h_{j}^{+}$in $L^{\infty}(0, T)$.

(ii) We only prove the last inequality in (4.5). Since $u$ is a solution of $(P)$ in $I_{j+1} \times(0, \tau)$, by (3.1) there holds

$$
\int_{0}^{\tau} \int_{I_{j+1}}\left\{\left(u_{r}-k\right) \xi_{t}+\left[\varphi\left(u_{r}\right)-\varphi(k)\right] \xi_{x}\right\} d x d t=-\int_{I_{j+1}}\left[u_{0 r}(x)-k\right] \xi(x, 0) d x
$$

for all $k \in[0, \infty)$ and $\xi \in C^{1}\left([0, \tau] ; C_{c}^{1}\left(I_{j+1}\right)\right), \xi(\cdot, \tau)=0$ in $I_{j+1}$. Let

$$
\eta_{\epsilon}(x):=\frac{2\left(x-x_{j}\right)-\epsilon}{\epsilon} \chi_{\left[x_{j}+\epsilon / 2, x_{j}+\epsilon\right]}(x)+\chi_{\left(x_{j}+\epsilon, x_{j+1}\right]}(x) \quad\left(x \in I_{j+1}\right)
$$

and let $\zeta \in C^{1}\left([0, \tau] ; C_{c}^{1}\left(\left[x_{j}, x_{j+1}\right)\right)\right), \zeta(\cdot, \tau)=0$ in $I_{j+1}$ (here $x_{j+1}=\infty$ if $\left.j=p\right)$. By standard arguments we can choose $\xi=\zeta \eta_{\epsilon}$ in the above equality, and obtain

$$
\begin{aligned}
& \int_{0}^{\tau} \int_{I_{j+1}}\left\{\left(u_{r}-k\right) \zeta_{t} \eta_{\epsilon}+\left[\varphi\left(u_{r}\right)-\varphi(k)\right] \zeta_{x} \eta_{\epsilon}\right\} d x d t+\int_{I_{j+1}}\left[u_{0 r}(x)-k\right] \zeta(x, 0) \eta_{\epsilon}(x) d x= \\
= & -\frac{2}{\epsilon} \int_{0}^{\tau} \int_{x_{j}+\epsilon / 2}^{x_{j}+\epsilon}\left[\varphi\left(u_{r}\right)-\varphi(k)\right] \zeta d x d t .
\end{aligned}
$$

Letting $\epsilon \rightarrow 0^{+}$in the above equality plainly gives (see (4.6)):

mkjn1

$$
\begin{aligned}
& \int_{0}^{\tau} \int_{I_{j+1}}\left\{\left(u_{r}-k\right) \zeta_{t}+\left[\varphi\left(u_{r}\right)-\varphi(k)\right] \zeta_{x}\right\} d x d t+\int_{I_{j+1}}\left[u_{0 r}(x)-k\right] \zeta(x, 0) d x= \\
= & - \text { ess } \lim _{x \rightarrow x_{j}^{+}} \int_{0}^{\tau}\left[\varphi\left(u_{r}(x, t)\right)-\varphi(k)\right] \zeta(x, t) d t=-\int_{0}^{\tau}\left[h_{j}^{+}(t)-\varphi(k)\right] \zeta\left(x_{j}, t\right) d t
\end{aligned}
$$

Since $u$ is an entropy solution of $(P)$ in $I_{j+1} \times(0, \tau)$, arguing as before we obtain

$$
\begin{aligned}
& \int_{0}^{\tau} \int_{I_{j+1}}\left\{\left|u_{r}-k\right| \zeta_{t}+\operatorname{sgn}\left(u_{r}-k\right)\left[\varphi\left(u_{r}\right)-\varphi(k)\right] \zeta_{x}\right\} d x d t+\int_{I_{j+1}}\left|u_{0 r}(x)-k\right| \zeta(x, 0) d x \geq \\
\geq & - \text { ess } \lim _{x \rightarrow x_{j}^{+}} \int_{0}^{\tau} \operatorname{sgn}\left(u_{r}(x, t)-k\right)\left[\varphi\left(u_{r}(x, t)\right)-\varphi(k)\right] \zeta(x, t) d t
\end{aligned}
$$


for all $\zeta$ as above, $\zeta \geq 0$. Choosing $\zeta(x, t)=\alpha(x) \beta(t)$ with $\alpha \in C_{c}^{1}\left(\left[x_{j}, x_{j+1}\right)\right), \alpha \geq 0$ and $\beta \in C^{1}([0, \tau]), \beta \geq 0, \beta(\tau)=0$, by the compatibility condition (3.7) there holds:

mkjn3

$$
\begin{aligned}
& \int_{0}^{\tau} \int_{I_{j+1}}\left\{\left|u_{r}-k\right| \zeta_{t}+\operatorname{sgn}\left(u_{r}-k\right)\left[\varphi\left(u_{r}\right)-\varphi(k)\right] \zeta_{x}\right\} d x d t+ \\
+ & \int_{I_{j+1}}\left|u_{0 r}(x)-k\right| \zeta(x, 0) d x+\operatorname{ess} \lim _{x \rightarrow x_{j}^{+}} \int_{0}^{\tau}\left[\varphi\left(u_{r}(x, t)\right)-\varphi(k)\right] \zeta(x, t) d t \geq \\
\geq & -2 \text { ess } \lim _{x \rightarrow x_{j}^{+}} \int_{0}^{\tau} H_{-}\left(u_{r}(x, t)-k\right)\left[\varphi\left(u_{r}(x, t)\right)-\varphi(k)\right] \zeta(x, t) d t= \\
= & -2 \alpha\left(x_{j}\right) \text { ess } \lim _{x \rightarrow x_{j}^{+}} \int_{0}^{\tau} H_{-}\left(u_{r}(x, t)-k\right)\left[\varphi\left(u_{r}(x, t)\right)-\varphi(k)\right] \beta(t) d t \geq 0,
\end{aligned}
$$

since $\operatorname{sgn}(u)=1+2 H_{-}(u)$. From inequalities (4.8) and (4.9) we obtain

$$
\begin{aligned}
& \int_{0}^{\tau} \int_{I_{j+1}}\left\{\left[u_{r}-k\right]_{+} \zeta_{t}+H_{+}\left(u_{r}-k\right)\left[\varphi\left(u_{r}\right)-\varphi(k)\right] \zeta_{x}\right\} d x d t+ \\
+ & \int_{I_{j+1}}\left[u_{0 r}(x)-k\right]_{+} \zeta(x, 0) d x \geq-\int_{0}^{\tau}\left[h_{j}^{+}(t)-\varphi(k)\right] \zeta\left(x_{j}, t\right) d t .
\end{aligned}
$$

Letting $k \rightarrow \infty$ in the above inequality gives

$$
\liminf _{k \rightarrow \infty} \int_{0}^{\tau}\left[h_{j}^{+}(t)-\varphi(k)\right] \zeta\left(x_{j}, t\right) d t=\int_{0}^{\tau}\left[h_{j}^{+}(t)-\limsup _{k \rightarrow \infty} \varphi(k)\right] \zeta\left(x_{j}, t\right) d t \geq 0,
$$

whence the last inequality in (4.5) follows by the arbitrariness of $\zeta$.

Replacing $I_{j+1} \times(0, \tau)$ by $I_{j} \times(0, \tau)$, we obtain, similarly to (4.8) and (4.9),

mkjn2

$$
\begin{aligned}
& \quad \int_{0}^{\tau} \int_{I_{j}}\left\{\left(u_{r}-k\right) \zeta_{t}+\left[\varphi\left(u_{r}\right)-\varphi(k)\right] \zeta_{x}\right\} d x d t+\int_{I_{j}}\left[u_{0 r}(x)-k\right] \zeta(x, 0) d x= \\
& =\text { ess } \lim _{x \rightarrow x_{j}^{-}} \int_{0}^{\tau}\left[\varphi\left(u_{r}(x, t)\right)-\varphi(k)\right] \zeta(x, t) d t=\int_{0}^{\tau}\left[h_{j}^{-}(t)-\varphi(k)\right] \zeta\left(x_{j}, t\right) d t,
\end{aligned}
$$

mkjn4

$$
\begin{aligned}
& \int_{0}^{\tau} \int_{I_{j}}\left\{\left|u_{r}-k\right| \zeta_{t}+\operatorname{sgn}\left(u_{r}-k\right)\left[\varphi\left(u_{r}\right)-\varphi(k)\right] \zeta_{x}\right\} d x d t+ \\
+ & \int_{I_{j}}\left|u_{0 r}(x)-k\right| \zeta(x, 0) d x-\operatorname{ess} \lim _{x \rightarrow x_{j}^{-}} \int_{0}^{\tau}\left[\varphi\left(u_{r}(x, t)\right)-\varphi(k)\right] \zeta(x, t) d t \geq 0,
\end{aligned}
$$

whence

$$
\begin{aligned}
& \int_{0}^{\tau} \int_{I_{j}}\left\{\left[u_{r}-k\right]_{+} \zeta_{t}+H_{+}\left(u_{r}-k\right)\left[\varphi\left(u_{r}\right)-\varphi(k)\right] \zeta_{x}\right\} d x d t+ \\
+ & \int_{I_{j}}\left[u_{0 r}(x)-k\right]_{+} \zeta(x, 0) d x \geq \int_{0}^{\tau}\left[h_{j}^{-}(t)-\varphi(k)\right] \zeta\left(x_{j}, t\right) d t
\end{aligned}
$$

and

$$
\limsup _{k \rightarrow \infty} \int_{0}^{\tau}\left[h_{j}^{-}(t)-\varphi(k)\right] \zeta\left(x_{j}, t\right) d t=\int_{0}^{\tau}\left[h_{j}^{-}(t)-\liminf _{k \rightarrow \infty} \varphi(k)\right] \zeta\left(x_{j}, t\right) d t \leq 0 .
$$

Since $\zeta$ is arbitrary we obtain the first inequality in (4.5).

Remark 4.2. By standard density arguments and (4.6), it follows from (4.11) that

nefo

$$
\begin{aligned}
& \int_{0}^{\tau} \int_{I_{1}}\left\{\left|u_{r}-k\right| \zeta_{t}+\operatorname{sgn}\left(u_{r}-k\right)\left[\varphi\left(u_{r}\right)-\varphi(k)\right] \zeta_{x}\right\} d x d t+ \\
+ & \int_{I_{1}}\left|u_{0 r}(x)-k\right| \zeta(x, 0) d x \geq \int_{0}^{\tau}\left[h_{1}^{-}(t)-\varphi(k)\right] \zeta\left(x_{1}, t\right) d t
\end{aligned}
$$


if $\zeta \in C^{1}\left([0, \tau] ; C_{c}^{1}\left(\left(-\infty, x_{1}\right]\right)\right), \zeta \geq 0, \zeta(\cdot, \tau)=0$ in $\left(-\infty, x_{1}\right]$, and from (4.9) that

$$
\begin{aligned}
& \int_{0}^{\tau} \int_{I_{p+1}}\left\{\left|u_{r}-k\right| \zeta_{t}+\operatorname{sgn}\left(u_{r}-k\right)\left[\varphi\left(u_{r}\right)-\varphi(k)\right] \zeta_{x}\right\} d x d t+ \\
+ & \int_{I_{p+1}}\left|u_{0 r}(x)-k\right| \zeta(x, 0) d x \geq-\int_{0}^{\tau}\left[h_{p}^{+}(t)-\varphi(k)\right] \zeta\left(x_{p}, t\right) d t
\end{aligned}
$$

for all $\zeta \in C^{1}\left([0, \tau] ; C_{c}^{1}\left(\left[x_{p}, \infty\right)\right)\right), \zeta \geq 0, \zeta(\cdot, \tau)=0$ in $\left[x_{p}, \infty\right)$. Moreover, arguing as in the proof of Lemma 4.4 with $\eta_{\epsilon}$ in (4.7) replaced by

$$
\frac{2\left(x-x_{j}\right)-\epsilon}{\epsilon} \chi_{\left[x_{j}+\epsilon / 2, x_{j}+\epsilon\right]}+\chi_{\left[x_{j}+\epsilon, x_{j+1}-\epsilon\right]}+\frac{2\left(x_{j+1}-x\right)-\epsilon}{\epsilon} \chi_{\left[x_{j+1}-\epsilon, x_{j+1}-\epsilon / 2\right]},
$$

we obtain that, for any $j=1, \ldots, p-1$,

$$
\begin{aligned}
& \int_{0}^{\tau} \int_{I_{j+1}}\left\{\left|u_{r}-k\right| \zeta_{t}+\operatorname{sgn}\left(u_{r}-k\right)\left[\varphi\left(u_{r}\right)-\varphi(k)\right] \zeta_{x}\right\} d x d t+\int_{I_{j+1}}\left|u_{0 r}(x)-k\right| \zeta(x, 0) d x \geq \\
\geq & -\int_{0}^{\tau}\left[h_{j}^{+}(t)-\varphi(k)\right] \zeta\left(x_{j}, t\right) d t+\int_{0}^{\tau}\left[h_{j+1}^{-}(t)-\varphi(k)\right] \zeta\left(x_{j+1}, t\right) d t
\end{aligned}
$$

for all $\zeta \in C^{1}\left([0, \tau] ; C_{c}^{1}\left(\left[x_{j}, x_{j+1}\right]\right)\right), \zeta \geq 0, \zeta(\cdot, \tau)=0$ in $\left[x_{j}, x_{j+1}\right]$.

mer Remark 4.3. For further reference we mention the following inequalities, which hold for all $\zeta \in C^{1}\left([0, \tau] ; C_{c}^{1}\left(\left(-\infty, x_{1}\right]\right)\right), \zeta \geq 0, \zeta(\cdot, \tau)=0$ in $\left(-\infty, x_{1}\right]$ :

subnefo

$$
\begin{aligned}
& \int_{0}^{\tau} \int_{I_{1}}\left\{\left[u_{r}-k\right]_{+} \zeta_{t}+H_{+}\left(u_{r}-k\right)\left[\varphi\left(u_{r}\right)-\varphi(k)\right] \zeta_{x}\right\} d x d t+ \\
+ & \int_{I_{1}}\left[u_{0 r}(x)-k\right]_{+} \zeta(x, 0) d x \geq \int_{0}^{\tau}\left[h_{1}^{-}(t)-\varphi(k)\right] \zeta\left(x_{1}, t\right) d t, \\
& \int_{0}^{\tau} \int_{I_{1}}\left\{\left[u_{r}-k\right]_{-} \zeta_{t}+H_{-}\left(u_{r}-k\right)\left[\varphi\left(u_{r}\right)-\varphi(k)\right] \zeta_{x}\right\} d x d t+ \\
+ & \int_{I_{1}}\left[u_{0 r}(x)-k\right]_{-} \zeta(x, 0) d x \geq 0 .
\end{aligned}
$$

The proof is analogous to that of (4.12), starting from (3.3) and (3.4) instead of (3.2). Similar inequalities hold in $S_{j}$ for $j=2, \ldots, p+1$ (see Remark 3.1).

4.2. Auxiliary problems. Let $j=1, \ldots, p+1$ and $n \in \mathbb{N}$. We consider the family of auxiliary problems

$$
\left(P_{j, n}\right) \quad \begin{cases}u_{t}+[\varphi(u)]_{x}=0 & \text { in } S_{j} \\ u=n & \text { in } \partial I_{j} \times(0, T) \\ u=u_{0 n}:=\min \left\{u_{0 r}, n\right\} & \text { in } I_{j} \times\{0\} .\end{cases}
$$

We follow [9, 10] to define entropy solutions of $\left(P_{j, n}\right)$.

otto Definition 4.1. By an entropy solution of problem $\left(P_{j, n}\right)$ we mean a function $u_{j, n} \in C\left([0, T] ; L_{l o c}^{1}\left(I_{j}\right)\right) \cap L^{\infty}\left(S_{j}\right)$ such that:

(i) $u_{j, n}$ is an entropy solution of problem $(P)$ in $S_{j}$ (in the sense of Definition 3.2) with Cauchy data $u_{0 n}$;

(ii) for all $\beta \in C_{c}^{1}(0, T), \beta \geq 0, k \in[0, \infty)$ and $n \geq k$

dienzu (4.15)

$$
\begin{aligned}
& \underset{x \rightarrow x_{j-1}^{+}}{\operatorname{ess}} \int_{0}^{T} H_{-}\left(u_{j, n}(x, t)-k\right)\left[\varphi\left(u_{j, n}(x, t)\right)-\varphi(k)\right] \beta(t) d t \leq 0 \quad \text { if } 2 \leq j \leq p+1, \\
& \operatorname{ess} \lim _{x \rightarrow x_{j}^{-}} \int_{0}^{T} H_{-}\left(u_{j, n}(x, t)-k\right)\left[\varphi\left(u_{j, n}(x, t)\right)-\varphi(k)\right] \beta(t) d t \geq 0 \quad \text { if } 1 \leq j \leq p .
\end{aligned}
$$


Remark 4.4. By Definitions 3.1 3.2 and 4.1, for all $\zeta \in C^{1}\left([0, T] ; C_{c}^{1}\left(I_{j}\right)\right), j, n$ and $k$ as above $u_{j, n}$ satisfies

$$
\iint_{S_{j}}\left[u_{j, n} \zeta_{t}+\varphi\left(u_{j, n}\right) \zeta_{x}\right] d x d t=-\int_{I_{j}} u_{0 n}(x) \zeta(x, 0) d x,
$$

mkrun (4.17)

$$
\iint_{S_{j}}\left\{\left|u_{j, n}-k\right| \zeta_{t}+\operatorname{sgn}\left(u_{j, n}-k\right)\left[\varphi\left(u_{j, n}\right)-\varphi(k)\right] \zeta_{x}\right\} d x d t \geq-\int_{I_{j}}\left|u_{0 n}(x)-k\right| \zeta(x, 0) d x
$$

By (3.4) and Remark 3.1, there also holds

superkrun (4.18)

$$
\iint_{S_{j}}\left\{\left[u_{j, n}-k\right]_{-} \zeta_{t}+H_{-}\left(u_{j, n}-k\right)\left[\varphi\left(u_{j, n}\right)-\varphi(k)\right] \zeta_{x}\right\} d x d t \geq-\int_{I_{j}}\left[u_{0 n}(x)-k\right]_{-} \zeta(x, 0) d x
$$

for all $\zeta \in C^{1}\left([0, \tau] ; C_{c}^{1}(\Omega)\right), \zeta \geq 0, \zeta(\cdot, \tau)=0$ in $\Omega$, and $k \in[0, \infty)$.

pe1 Proposition 4.5. Let $\left(H_{0}\right)-\left(H_{1}\right)$ hold. Then for all $j=1, \ldots, p+1$ there exists an entropy solution $u_{j} \in C\left([0, T] ; L_{l o c}^{1}\left(I_{j}\right)\right)$ of problem $(P)$ in $S_{j}$, such that for all $k \geq 0$

dienzz

$$
\begin{aligned}
& \text { ess } \lim _{x \rightarrow x_{j}^{+}} \int_{0}^{T} H_{-}\left(u_{j+1}(x, t)-k\right)\left[\varphi\left(u_{j+1}(x, t)\right)-\varphi(k)\right] \beta(t) d t \leq 0, \\
& \text { ess } \lim _{x \rightarrow x_{j}^{-}} \int_{0}^{T} H_{-}\left(u_{j}(x, t)-k\right)\left[\varphi\left(u_{j}(x, t)\right)-\varphi(k)\right] \beta(t) d t \geq 0 .
\end{aligned}
$$

Moreover, $u_{j} \in C\left([0, T] ; L^{1}\left(I_{j}\right)\right)$ for $j=2, \ldots, p$.

According to 9, 10], if $\varphi \in C^{2}([0, \infty))$ for every $j=1, \ldots, p+1, n \in \mathbb{N}$ there exists a unique entropy solution $u_{j, n}$ of $\left(P_{j, n}\right)$. To prove Proposition 4.5 we need some preliminary results about these solutions.

pe2 Lemma 4.6. Let $\left(H_{1}\right)$ hold, and let $u_{j, n}$ be the unique entropy solution of $\left(P_{j, n}\right)$ $(j=1, \ldots, p+1 ; n \in \mathbb{N})$. Then:

(i) there holds $0 \leq u_{j, n} \leq n, u_{j, n} \leq u_{j, n+1}$ a.e. in $S_{j}$;

(ii) the sequence $\left\{u_{j, n}\right\}$ is bounded in $C\left([0, T] ; L^{1}\left(I_{j}\right)\right)$ if $j=2, \ldots, p,\left\{u_{1, n}\right\}$ is bounded in $C\left([0, T] ; L_{l o c}^{1}\left(I_{1}\right)\right)$, and $\left\{u_{p+1, n}\right\}$ is bounded in $C\left([0, T] ; L_{l o c}^{1}\left(I_{p+1}\right)\right)$.

Proof. (i) We only give the proof if $j=1$. Consider the problems

$$
\begin{cases}u_{t}+[\varphi(u)]_{x}=0 & \text { in } S_{1} \\ u=a_{i} & \text { in }\left\{x_{1}\right\} \times(0, T) \\ u=b_{i} & \text { in } I_{1} \times\{0\},\end{cases}
$$

where $a_{i} \in L^{\infty}(0, T), b_{i} \in L^{\infty}\left(I_{1}\right)(i=1,2)$. As already mentioned, for each $i$ there exists a unique entropy solution $z_{i} \in C\left([0, T] ; L_{l o c}^{1}\left(I_{1}\right)\right) \cap L^{\infty}\left(S_{1}\right)$ of $\left(P_{i}\right)$. Moreover, for every $\left(x_{0}, t\right) \in S_{1}$ there holds (see [9, 10])

ost (4.20)

$$
\int_{x_{0}}^{x_{1}}\left|z_{1}(x, t)-z_{2}(x, t)\right| d x \leq \int_{x_{0}-\left\|\varphi^{\prime}\right\|_{\infty} t}^{x_{1}}\left|b_{1}(x)-b_{2}(x)\right| d x+\left\|\varphi^{\prime}\right\|_{\infty} \int_{0}^{t}\left|a_{1}(s)-a_{2}(s)\right| d s .
$$

Consider four sequences $\left\{a_{i k}\right\} \subset B V_{l o c}(0, T),\left\{b_{i k}\right\} \subset B V_{l o c}\left(I_{1}\right)$ such that $a_{i k} \rightarrow a_{i}$ in $L_{l o c}^{1}(0, T), b_{i k} \rightarrow b_{i}$ in $L_{l o c}^{1}\left(I_{1}\right)$ as $k \rightarrow \infty(i=1,2)$. Let $z_{i k} \in B V_{l o c}\left(S_{1}\right)$ be the 
unique entropy solution of $\left(P_{i}\right)$ with boundary and initial data $a_{i k}, b_{i k}$. As proven in [14], for every $\left(x_{0}, t\right) \in S_{1}$ and $k \in \mathbb{N}$ there holds

$$
\begin{aligned}
\int_{x_{0}}^{x_{1}}\left[z_{1 k}(x, t)-z_{2 k}(x, t)\right]_{+} d x & \leq \int_{x_{0}-\left\|\varphi^{\prime}\right\|_{\infty} t}^{x_{1}}\left[b_{1 k}(x)-b_{2 k}(x)\right]_{+} d x+ \\
& +\left\|\varphi^{\prime}\right\|_{\infty} \int_{0}^{t}\left[a_{1 k}(s)-a_{2 k}(s)\right]_{+} d s
\end{aligned}
$$

On the other hand, applying (4.20) to $z_{1 k}$ and $z_{2 k}$, by the arbitrariness of $x_{0}$ we obtain that $z_{i k}(\cdot, t) \rightarrow z_{i}(\cdot, t)$ in $L_{l o c}^{1}\left(I_{1}\right)$ as $k \rightarrow \infty$, for all $t \in(0, T)$. Hence there exists a subsequence $z_{i k_{l}}(\cdot, t) \subseteq z_{i k}(\cdot, t)$ such that $z_{i k_{l}}(\cdot, t) \rightarrow z_{i}(\cdot, t)$ a.e. in $I_{1}$. Letting $k_{l} \rightarrow \infty$ in (4.21) (with $k=k_{l}$ ), we obtain from Fatou's Lemma that

$$
\begin{aligned}
\int_{x_{0}}^{x_{1}}\left[z_{1}(x, t)-z_{2}(x, t)\right]_{+} d x & \leq \int_{x_{0}-\left\|\varphi^{\prime}\right\|_{\infty} t}^{x_{1}}\left[b_{1}(x)-b_{2}(x)\right]_{+} d x+ \\
& +\left\|\varphi^{\prime}\right\|_{\infty} \int_{0}^{t}\left[a_{1}(s)-a_{2}(s)\right]_{+} d s
\end{aligned}
$$

whence the claim immediately follows.

(ii) Let $j \in\{2, \ldots, p\}$ be fixed. Choosing in (4.16) $\zeta(x, t)=\alpha(x) \beta(t)$ with $\alpha \epsilon$ $C_{c}^{1}\left(I_{j}\right), \alpha \geq 0$, and $\beta \in C^{1}([0, T]), \beta(T)=0$ we obtain

$$
\begin{aligned}
& \left|\int_{0}^{T} d t \beta^{\prime}(t) \int_{I_{j}} u_{j, n}(x, t) \alpha(x) d x\right| \leq\left|\int_{0}^{T} d t \beta(t) \int_{I_{j}} \varphi\left(u_{j, n}(x, t)\right) \alpha^{\prime}(x) d x\right|+ \\
+ & \left|\beta(0) \int_{I_{j}} u_{0 n}(x) \alpha(x) d x\right| \leq\|\beta\|_{\infty}\left(T\|\varphi\|_{\infty}+\left\|u_{0}\right\|_{L^{1}\left(I_{j}\right)}\right)\|\alpha\|_{W^{1,1}\left(I_{j}\right)} .
\end{aligned}
$$

By standard smoothing arguments we can set, for fixed $\tau \in(0, T), \beta=\beta_{m}$,

$$
\beta_{m}(t):=\chi_{(0, \tau]}(t)-m\left(t-\tau-\frac{1}{m}\right) \chi_{\left(\tau, \tau+\frac{1}{m}\right]}(t) \text { for } t \in[0, T],
$$

for sufficiently large $m \in \mathbb{N}$. Letting $m \rightarrow \infty$ gives for all $\tau \in(0, T)$

$$
0 \leq \int_{I_{j}} u_{j, n}(x, \tau) \alpha(x) d x \leq\|\beta\|_{\infty}\left(T\|\varphi\|_{\infty}+\left\|u_{0}\right\|_{L^{1}\left(I_{j}\right)}\right)\|\alpha\|_{W^{1,1}\left(I_{j}\right)} .
$$

We fix $\epsilon>0$ and choose $\alpha$ in (4.23) as

$m\left(x-x_{j-1}-\epsilon\right) \chi_{\left(x_{j-1}+\epsilon, x_{j-1}+\epsilon+\frac{1}{m}\right]}+\chi_{\left(x_{j-1}+\epsilon+\frac{1}{m}, x_{j}-\epsilon-\frac{1}{m}\right]}-m\left(x-x_{j}+\epsilon\right) \chi_{\left(x_{j}-\epsilon-\frac{1}{m}, x_{j}-\epsilon\right]}$.

Letting $m \rightarrow \infty$ we obtain that

$$
0 \leq \int_{x_{j-1}+\epsilon}^{x_{j}-\epsilon} u_{j, n}(x, \tau) d x \leq\|\beta\|_{\infty}\left(T\|\varphi\|_{\infty}+\left\|u_{0}\right\|_{L^{1}\left(I_{j}\right)}\right)\left(x_{j}-x_{j-1}-2 \epsilon+2\right),
$$

whence, by the arbitrariness of $\epsilon$,

$$
0 \leq \int_{I_{j}} u_{j, n}(x, \tau) d x \leq\|\beta\|_{\infty}\left(T\|\varphi\|_{\infty}+\left\|u_{0}\right\|_{L^{1}\left(I_{j}\right)}\right)\left(x_{j}-x_{j-1}+2\right) .
$$

This completes the proof if $j=2, \ldots, p$. A similar argument can be used in bounded subsets of $S_{1}$ and $S_{p+1}$, hence the conclusion follows.

Proof of Proposition 4.5. By Lemma 4.6 $(i)$ we may define

$$
u_{j}(x, t):=\lim _{n \rightarrow \infty} u_{j, n}(x, t) \text { for a.e. }(x, t) \in S_{j} .
$$

Let $n \rightarrow \infty$ in (4.24). By monotonicity, $u_{j} \in L^{\infty}\left(0, T ; L^{1}\left(I_{j}\right)\right)$ and

$$
u_{j, n} \rightarrow u_{j} \quad \text { in } L^{1}\left(S_{j}\right)
$$


for $j=2, \ldots, p$. Similarly, $u_{1} \in L^{\infty}\left(0, T ; L_{l o c}^{1}\left(I_{1}\right)\right), u_{p+1} \in L^{\infty}\left(0, T ; L_{l o c}^{1}\left(I_{p+1}\right)\right)$ and

$$
u_{1, n} \rightarrow u_{1} \text { in } L_{l o c}^{1}\left(S_{1}\right), \quad u_{p+1, n} \rightarrow u_{p+1} \text { in } L_{l o c}^{1}\left(S_{p+1}\right) .
$$

From the above convergences, letting $j \rightarrow \infty$ in (4.16) and (4.17) we easily get

wefoj

$$
\iint_{S_{j}}\left[u_{j} \zeta_{t}+\varphi\left(u_{j}\right) \zeta_{x}\right] d x d t=-\int_{I_{j}} u_{0 r}(x) \zeta(x, 0) d x,
$$

mkruj

$$
\iint_{S_{j}}\left\{\left|u_{j}-k\right| \zeta_{t}+\operatorname{sgn}\left(u_{j}-k\right)\left[\varphi\left(u_{j}\right)-\varphi(k)\right] \zeta_{x}\right\} d x d t \geq-\int_{I_{j}}\left|u_{0 r}(x)-k\right| \zeta(x, 0) d x
$$

for all $\zeta \in C^{1}\left([0, \tau] ; C_{c}^{1}\left(I_{j}\right)\right), \zeta \geq 0, \zeta(\cdot, \tau)=0$ in $I_{j}$.

Next we show that $u_{j} \in C\left([0, T] ; L^{1}\left(I_{j}\right)\right)$ for every $j=2, \ldots, p$ (the same argument shows that $u_{1} \in C\left([0, T] ; L_{l o c}^{1}\left(I_{1}\right)\right)$ and $\left.u_{p+1} \in C\left([0, T] ; L_{l o c}^{1}\left(I_{p+1}\right)\right)\right)$. By 3. Proposition 3.10] and the above remarks there holds $u_{j} \in C\left((0, T] ; L^{1}\left(I_{j}\right)\right)$. To prove the continuity at $t=0$, observe that for any $\alpha \in C_{c}^{1}\left(I_{j}\right), \alpha \geq 0$ and $h$ sufficiently small

$$
\begin{aligned}
\text { (4.30) } & \int_{I_{j}}\left|u_{j, n}(x, \tau)-u_{j, n}(x+h, \tau)\right| \alpha(x) d x \leq \int_{I_{j}}\left|u_{0 n}(x)-u_{0 n}(x+h)\right| \alpha(x) d x+ \\
+ & \int_{0}^{\tau} \int_{I_{j}}\left|\varphi\left(u_{j, n}(x, t)\right)-\varphi\left(u_{j, n}(x+h, t)\right)\right|\left|\alpha^{\prime}(x)\right| d x d t
\end{aligned}
$$

for all $\tau \in(0, T)$ (the above inequality derives from the $L^{1}$-contraction property of the parabolic equation satisfied by the parabolic approximants of $u_{j, n}$; see [9, 10]).

By (4.26)-(4.27), as $n \rightarrow \infty$ in (4.30) we obtain for all $\tau \in(0, T)$

$$
\begin{aligned}
& \int_{I_{l}}\left|u_{j}(x, \tau)-u_{j}(x+h, \tau)\right| \alpha(x) d x \leq \int_{I_{l}}\left|u_{0 r}(x)-u_{0 r}(x+h)\right| \alpha(x) d x+ \\
+ & \int_{0}^{\tau} \int_{I_{l}}\left|\varphi\left(u_{j}(x, t)\right)-\varphi\left(u_{j}(x+h, t)\right)\right|\left|\alpha^{\prime}(x)\right| d x d t .
\end{aligned}
$$

Let $\left\{\tau_{k}\right\} \subset(0, T), \tau_{k} \rightarrow 0^{+}$as $k \rightarrow \infty$. Since $u_{0 r} \in L^{1}\left(I_{j}\right)$ and $\varphi\left(u_{j}\right) \in L^{1}\left(S_{j}\right)$, by (4.31) and the Fréchet-Kolmogorov Theorem the sequence $\left\{u_{j}\left(\cdot, \tau_{k}\right) \alpha\right\}$ is relatively compact in $L^{1}\left(I_{j}\right)$. Then by (4.28) and a standard argument there holds $u_{j}\left(\cdot, \tau_{k}\right) \alpha \rightarrow u_{0 r} \alpha$ in $L^{1}\left(I_{j}\right)$ as $k \rightarrow \infty$. Arguing as in the proof of [3. Proposition 3.10] we obtain that $\lim _{k \rightarrow \infty} \int_{I_{j}}\left|u_{j}\left(x, \tau_{k}\right)-u_{0 r}(x)\right| d x=0$, so $u_{j} \in C\left([0, T] ; L^{1}\left(I_{j}\right)\right)$.

It remains to prove (4.19). We only prove (4.19) 1 . By (4.25) and the Dominated Convergence Theorem, for a.e. $x \in I_{j}$

$$
\begin{aligned}
& \lim _{n \rightarrow \infty} \int_{0}^{T} H_{-}\left(u_{j, n}(x, t)-k\right)\left[\varphi\left(u_{j, n}(x, t)\right)-\varphi(k)\right] \beta(t) d t= \\
= & \int_{0}^{T} H_{-}\left(u_{j}(x, t)-k\right)\left[\varphi\left(u_{j}(x, t)\right)-\varphi(k)\right] \beta(t) d t .
\end{aligned}
$$

Then for a.e. $x \in I_{j}$ and every $\epsilon>0$ there exists $\bar{n}=\bar{n}(x) \geq k$ such that

$$
\begin{aligned}
& \int_{0}^{T} H_{-}\left(u_{j}(x, t)-k\right)\left[\varphi\left(u_{j}(x, t)\right)-\varphi(k)\right] \beta(t) d t \leq \\
\leq & \int_{0}^{T} H_{-}\left(u_{j, \bar{n}}(x, t)-k\right)\left[\varphi\left(u_{j, \bar{n}}(x, t)\right)-\varphi(k)\right] \beta(t) d t+\epsilon .
\end{aligned}
$$

On the other hand, arguing as in the proof of Lemma 4.2. (4.18) implies that

$$
x \mapsto \int_{0}^{T} H_{-}\left(u_{j, n}(x, t)-k\right)\left[\varphi\left(u_{j, n}(x, t)\right)-\varphi(k)\right] \beta(t) d t-k T\left\|\beta^{\prime}\right\|_{\infty} x
$$


is nonincreasing in $\mathbb{R}$. Then by (4.15) 1 we get that for a.e. $x \in I_{j}$ and $n \geq k$

$$
\int_{0}^{T} H_{-}\left(u_{j, n}(x, t)-k\right)\left[\varphi\left(u_{j, n}(x, t)\right)-\varphi(k)\right] \beta(t) d t \leq k T\left\|\beta^{\prime}\right\|_{\infty}\left(x-x_{j-1}\right) .
$$

By (4.32)-(4.33) and the arbitrariness of $\epsilon$, for a.e. $x \in I_{j}$ we obtain

$$
\int_{0}^{T} H_{-}\left(u_{j}(x, t)-k\right)\left[\varphi\left(u_{j}(x, t)\right)-\varphi(k)\right] \beta(t) d t \leq k T\left\|\beta^{\prime}\right\|_{\infty}\left(x-x_{j-1}\right),
$$

whence 4.19) 1 follows.

4.3. Existence proof. Now we can prove Theorem 4.1

Proof of Theorem 4.1. Let $u_{j} \in C\left([0, T] ; L^{1}\left(I_{j}\right)\right)(2 \leq j \leq p), u_{1} \in C\left([0, T] ; L_{l o c}^{1}\left(I_{1}\right)\right)$ and $u_{p+1} \in C\left([0, T] ; L_{l o c}^{1}\left(I_{p+1}\right)\right)$ be given by Proposition 4.5 , and let $h_{j}^{ \pm}$be given by Lemma 4.4. For $j=1, \ldots, p$ we set

$$
C_{j}(t):=\left[c_{j}-\int_{0}^{t}\left[h_{j}^{+}(s)-h_{j}^{-}(s)\right] d s\right]_{+} \quad(t \in[0, T]) .
$$

Let $\bar{t}_{j}:=\sup \left\{\tau \in[0, T] \mid C_{j}(\tau)>0\right\}>0$. Then $\bar{t}_{j}>0$ since $C_{j}(0)=c_{j}>0$. By (4.5), $C_{j}$ is nonincreasing in $(0, T)$, whence $C_{j}>0$ in $\left[0, \bar{t}_{j}\right)$ and, if $\bar{t}_{j}<T, C_{j}=0$ in $\left[\bar{t}_{j}, T\right]$ (observe that $\bar{t}_{j}=t_{j}$ for every $j=1, \ldots, p$, with $t_{j}$ given by (3.6)). Let $\tau_{1}:=\min \left\{\bar{t}_{1}, \ldots, \bar{t}_{p}\right\}$, and define $u \in C\left(\left[0, \tau_{1}\right] ; \mathcal{M}^{+}(\mathbb{R})\right)$ by setting

$$
\left\{\begin{array}{l}
u_{r}(\cdot, t):=u_{j}(\cdot, t) \text { in } I_{j} \quad(j=1, \ldots, p+1) \\
u_{s}(\cdot, t):=\sum_{j=1}^{p} C_{j}(t) \delta_{x_{j}}
\end{array} \quad \text { for } 0 \leq t \leq \tau_{1} .\right.
$$

It follows from Proposition 4.5 that $u$ is an entropy solution of $(P)$ in $I_{j} \times\left(0, \tau_{1}\right)$ for $j=1, \ldots, p+1$ which satisfies the compatibility condition at every $x_{1}, \ldots, x_{p}$ in $\left[0, \tau_{1}\right]$. Hence $u$ is an entropy solution of $(P)$ in $\mathbb{R} \times\left(0, \tau_{1}\right)$, if we prove (3.1)-(3.2) with $\Omega=\mathbb{R}, \tau=\tau_{1}$ for all $\zeta \in C^{1}\left(\left[0, \tau_{1}\right] ; C_{c}^{1}(\mathbb{R})\right), \zeta \geq 0, \zeta\left(\cdot, \tau_{1}\right)=0$ in $\mathbb{R}$, such that

$$
\operatorname{supp} \zeta \cap\left(\left\{x_{j}\right\} \times\left(0, \tau_{1}\right)\right) \neq \varnothing \quad \text { for some } j=1, \ldots, p \text {. }
$$

We only give the proof when $\zeta(x, t)=\alpha(x) \beta(t)$ with $\alpha \in C_{c}^{1}(\mathbb{R}), \alpha \geq 0, \alpha\left(x_{j}\right)>0$ for a unique $j \in\{1, \ldots, p\}$, and $\beta \in C^{1}\left(\left[0, \tau_{1}\right]\right), \beta \geq 0, \beta\left(\tau_{1}\right)=0$ (the general case can be dealt with similarly). Let us first prove (3.1) in this case, namely

$$
\begin{aligned}
& \int_{0}^{\tau_{1}} \int_{I_{j} \cup I_{j+1}}\left[u_{r} \zeta_{t}+\varphi\left(u_{r}\right) \zeta_{x}\right] d x d t+\int_{I_{j} \cup I_{j+1}} u_{0 r}(x) \zeta(x, 0) d x= \\
- & \int_{0}^{\tau}\left\langle u_{s}(\cdot, t), \zeta_{t}(\cdot, t)\right\rangle_{\left(x_{j-1}, x_{j+1}\right)} d t-\left\langle u_{0 s}, \zeta(\cdot, 0)\right\rangle_{\left(x_{j-1}, x_{j+1}\right)}
\end{aligned}
$$

for all $\zeta$ as above (we set $x_{0}:=-\infty, x_{p+1}:=\infty$ ). From (4.34) and (4.35) we obtain

$$
\begin{aligned}
& \int_{0}^{\tau_{1}}\left\langle u_{s}(\cdot, t), \zeta_{t}(\cdot, t)\right\rangle_{\left(x_{j-1}, x_{j+1}\right)} d t+\left\langle u_{0 s}, \zeta(\cdot, 0)\right\rangle_{\left(x_{j-1}, x_{j+1}\right)}= \\
= & \alpha\left(x_{j}\right)\left(\int_{0}^{\tau_{1}} \beta^{\prime}(t) C_{j}(t) d t+c_{j} \beta(0)\right)=\alpha\left(x_{j}\right) \int_{0}^{\tau_{1}}\left[h_{j}^{+}(t)-h_{j}^{-}(t)\right] \beta(t) d t
\end{aligned}
$$

(see Lemma 4.4). On the other hand, summing (4.8) and (4.10) with $\tau=\tau_{1}$ gives

$$
\begin{aligned}
& \int_{0}^{\tau_{1}} \int_{I_{j} \cup I_{j+1}}\left[u_{r} \zeta_{t}+\varphi\left(u_{r}\right) \zeta_{x}\right] d x d t+\int_{I_{j} \cup I_{j+1}} u_{0 r}(x) \zeta(x, 0) d x= \\
= & -\alpha\left(x_{j}\right) \int_{0}^{\tau_{1}}\left[h_{j}^{+}(t)-h_{j}^{-}(t)\right] \beta(t) d t .
\end{aligned}
$$

and (4.36) follows from (4.37) and (4.38). 
Next we prove (3.2) for all $\zeta$ as above, namely

mkrum

$$
\begin{aligned}
& \int_{0}^{\tau_{1}} \int_{I_{j} \cup I_{j+1}}\left\{\left|u_{r}-k\right| \zeta_{t}+\operatorname{sgn}\left(u_{r}-k\right)\left[\varphi\left(u_{r}\right)-\varphi(k)\right] \zeta_{x}\right\} d x d t+ \\
+ & \int_{0}^{\tau_{1}}\left\langle u_{s}(\cdot, t), \zeta_{t}(\cdot, t)\right\rangle_{\left(x_{j-1}, x_{j+1}\right)} d t+\left\langle u_{0 s}, \zeta(\cdot, 0)\right\rangle_{\left(x_{j-1}, x_{j+1}\right)} \geq \\
\geq & -\int_{I_{j} \cup I_{j+1}}\left|u_{0 r}(x)-k\right| \zeta(x, 0) d x
\end{aligned}
$$

Since $u$ is an entropy solution of $(P)$ in $I_{j} \times\left(0, \tau_{1}\right)$ and $I_{j+1} \times\left(0, \tau_{1}\right)$, and satisfies the compatibility condition (3.7) in [0, $\left.\tau_{1}\right]$, it follows from (4.9) and (4.11) that

$$
\begin{aligned}
& \int_{0}^{\tau_{1}} \int_{I_{j} \cup I_{j+1}}\left\{\left|u_{r}-k\right| \zeta_{t}+\operatorname{sgn}\left(u_{r}-k\right)\left[\varphi\left(u_{r}\right)-\varphi(k)\right] \zeta_{x}\right\} d x d t+ \\
+ & \int_{I_{j} \cup I_{j+1}}\left|u_{0 r}(x)-k\right| \zeta(x, 0) d x \geq-\alpha\left(x_{j}\right) \int_{0}^{\tau_{1}}\left[h_{j}^{+}(t)-h_{j}^{-}(t)\right] \beta(t) d t .
\end{aligned}
$$

Combined with (4.37) this implies (4.39). Therefore, the measure $u$ defined by (4.35) is an entropy solution of $(P)$ in $\mathbb{R} \times\left(0, \tau_{1}\right)$.

If $\tau_{1}<T$, either $u_{s}\left(\cdot, \tau_{1}\right)=0$, or $u_{s}\left(\cdot, \tau_{1}\right)>0$. If $u_{s}\left(\cdot, \tau_{1}\right)=0$, there holds $C_{j}\left(\tau_{1}\right)=0$ for all $j=1, \ldots, p$ (see (4.34)-(4.35) ), thus $u_{s}(\cdot, t)=0$ for all $t \in\left[\tau_{1}, T\right]$. Then, by the standard theory of scalar conservation laws, we can continue the solution of $(P)$ in $\left(\tau_{1}, T\right]$ with initial data $u_{r}\left(\cdot, \tau_{1}\right)$. On the other hand, if $u_{s}\left(\cdot, \tau_{1}\right)>0$, then $C_{j}\left(\tau_{1}\right)>0$ for some $j=1, \ldots, p$ and, arguing as before, we can continue the solution of $(P)$ in $\left(\tau_{1}, \tau_{2}\right]$, with initial data $u\left(\cdot, \tau_{1}\right)$, for some $\tau_{2} \in\left(\tau_{1}, T\right]$. Iterating the procedure $q$ times with $2 \leq q \leq p$, we obtain that either $\tau_{q}=T$, or $u_{s}\left(\cdot, \tau_{q}\right)=0$.

\section{Proof of UniQueness}

This section is devoted to the proof of the uniqueness part of Theorem 3.1

uniq Theorem 5.1. Let $\left(H_{0}\right)-\left(H_{1}\right)$ be satisfied. Then there exists at most one entropy solution of problem $(P)$, which belongs to $C\left([0, T] ; \mathcal{M}^{+}(\mathbb{R})\right)$ and satisfies the compatibility condition at $x_{j}$ in $\left[0, t_{j}\right]$ for all $j=1, \ldots, p$.

Proof. Let $u, v \in C\left([0, T] ; \mathcal{M}^{+}(\Omega)\right)$ be entropy solutions of $(P)$ satisfying the compatibility condition at every $x_{j}$ in $\left[0, t_{j}\right]$, and let

$$
\tau:=\min \left\{t_{u}, t_{v}\right\} \quad \text { where }\left\{\begin{array}{l}
t_{u}:=\sup \left\{t \in[0, T) \mid \operatorname{supp} u_{s}(\cdot, t)=\operatorname{supp} u_{0 s}\right\} \\
t_{v}:=\sup \left\{t \in[0, T) \mid \operatorname{supp} v_{s}(\cdot, t)=\operatorname{supp} u_{0 s}\right\} .
\end{array}\right.
$$

Arguing as at the end of the proof of Theorem 4.1, it is enough to show that

$$
u=v \quad \text { in } \mathcal{M}\left(S_{\tau}\right) .
$$

We claim that (5.2) follows if we prove that

$$
u_{r}=v_{r} \quad \text { a.e. in } \mathbb{R} \times(0, \tau)=: S_{\tau} .
$$

Indeed, (3.1) and (5.3) imply that, for all $\zeta \in C^{1}\left([0, \tau] ; C_{c}^{1}(\mathbb{R})\right), \zeta(\cdot, \tau)=0$ in $\mathbb{R}$,

$$
\int_{0}^{\tau}\left\langle u_{s}(\cdot, t)-v_{s}(\cdot, t), \zeta_{t}(\cdot, t)\right\rangle_{\mathbb{R}} d t=\iint_{S_{\tau}}\left\{\left(u_{r}-v_{r}\right) \zeta_{t}+\left[\varphi\left(u_{r}\right)-\varphi\left(u_{r}\right)\right] \zeta_{x}\right\} d x d t=0
$$

Hence $\left\langle u_{s}(\cdot, t)-v_{s}(\cdot, t), \alpha\right\rangle_{\mathbb{R}}=0$ for a.e. $t \in(0, \tau)$, for all $\alpha \in C_{c}^{1}(\mathbb{R})$. Therefore $u_{s}=v_{s}$ in $L^{\infty}(0, \tau ; \mathcal{M}(\mathbb{R}))$ and (5.2) follows from (5.3).

It remains to prove (5.3), which is equivalent to showing that

$$
u_{r}=v_{r} \quad \text { a.e. in } I_{j} \times(0, \tau) \text { for all } j=1, \ldots, p+1 \text {. }
$$


We only prove (5.4) for $j=1$, since in the other cases the proof is similar. Set $Q_{1}:=$ $\left(-\infty, x_{1}\right] \times(0, \tau)$. We apply the Kružkov method of doubling variables adapted to boundary valued problems (see $9,10,13)$. Let $\xi=\xi(x, t, y, s)$ be defined in $Q_{1} \times Q_{1}$, $\xi \geq 0$, such that $\xi(\cdot, \cdot, y, s) \in C_{c}^{1}\left(Q_{1}\right)$ for every $(y, s) \in Q_{1}$ and $\xi(x, t, \cdot, \cdot) \in C_{c}^{1}\left(Q_{1}\right)$ for every $(x, t) \in Q_{1}$. It follows from (4.12) that

$$
\begin{aligned}
& \iint_{Q_{1}}\left\{\operatorname{sgn}\left(u_{r}(x, t)-v(y, s)\right)\left[\varphi\left(u_{r}(x, t)\right)-\varphi\left(v_{r}(y, s)\right)\right] \xi_{x}(x, t, y, s)+\right. \\
+ & \left.\left|u_{r}(x, t)-v_{r}(y, s)\right| \xi_{t}(x, t, y, s)\right\} d x d t \geq \int_{0}^{\tau}\left[h_{1}^{-}(t)-\varphi\left(v_{r}(y, s)\right)\right] \xi\left(x_{1}, t, y, s\right) d t, \\
& \iint_{Q_{1}}\left\{\operatorname{sgn}\left(u_{r}(x, t)-v(y, s)\right)\left[\varphi\left(u_{r}(x, t)\right)-\varphi\left(v_{r}(y, s)\right)\right] \xi_{y}(x, t, y, s)+\right. \\
+ & \left.\left|u_{r}(x, t)-v_{r}(y, s)\right| \xi_{s}(x, t, y, s)\right\} d y d s \geq \int_{0}^{\tau}\left[g_{1}^{-}(s)-\varphi\left(u_{r}(x, t)\right)\right] \xi\left(x, t, x_{1}, s\right) d s,
\end{aligned}
$$

where, by Lemma $4.4(i), g_{j}^{ \pm} \in L^{\infty}(0, T)$ satisfies, for all $j=1, \ldots, p, g_{j}^{ \pm} \geq 0$ and

$$
\text { ess } \lim _{x \rightarrow x_{j}^{ \pm}} \int_{0}^{T} \varphi\left(v_{r}(x, t)\right) \beta(t) d t=\int_{0}^{T} g_{j}^{ \pm}(t) \beta(t) d t \quad \text { if } \beta \in L^{1}(0, T) .
$$

Let $\rho_{\epsilon}(\epsilon>0)$ be a symmetric mollifier in $\mathbb{R}$, and set in the previous inequalities

$$
\xi(x, t, y, s)=\eta\left(\frac{x+y}{2}, \frac{t+s}{2}\right) \rho_{\epsilon}(x-y) \rho_{\epsilon}(t-s)
$$

with $\eta \in C_{c}^{1}\left(\left(-\infty, x_{1}\right] \times(0, \tau)\right), \eta \geq 0$. Then we obtain

$$
\begin{aligned}
& \iiint \int_{Q_{1} \times Q_{1}} \rho_{\epsilon}(x-y) \rho_{\epsilon}(t-s)\left\{\left|u_{r}(x, t)-v_{r}(y, s)\right| \eta_{t}\left(\frac{x+y}{2}, \frac{t+s}{2}\right)+\right. \\
+ & \left.\operatorname{sgn}\left(u_{r}(x, t)-v_{r}(y, s)\right)\left[\varphi\left(u_{r}(x, t)\right)-\varphi\left(v_{r}(y, s)\right)\right] \eta_{x}\left(\frac{x+y}{2}, \frac{t+s}{2}\right)\right\} d x d t d y d s \geq \\
\geq & \int_{0}^{\tau} \iint_{Q_{1}}\left[g_{1}^{-}(s)-\varphi\left(u_{r}(x, t)\right)\right] \eta\left(\frac{x+x_{1}}{2}, \frac{t+s}{2}\right) \rho_{\epsilon}\left(x_{1}-x\right) \rho_{\epsilon}(t-s) d x d t d s+ \\
+ & \int_{0}^{\tau} \iint_{Q_{1}}\left[h_{1}^{-}(t)-\varphi\left(v_{r}(y, s)\right)\right] \eta\left(\frac{x_{1}+y}{2}, \frac{t+s}{2}\right) \rho_{\epsilon}\left(y-x_{1}\right) \rho_{\epsilon}(t-s) d y d s d t .
\end{aligned}
$$

Concerning the right-hand side of (5.7), by well-known properties of mollifiers

$$
\begin{gathered}
\int_{0}^{\tau} \iint_{Q_{1}} g_{1}^{-}(s) \eta\left(\frac{x+x_{1}}{2}, \frac{t+s}{2}\right) \rho_{\epsilon}\left(x_{1}-x\right) \rho_{\epsilon}(t-s) d x d t d s \rightarrow \frac{1}{2} \int_{0}^{\tau} g_{1}^{-}(s) \eta\left(x_{1}, s\right) d s, \\
\int_{0}^{\tau} \iint_{Q_{1}} h_{1}^{-}(t) \eta\left(\frac{x_{1}+y}{2}, \frac{t+s}{2}\right) \rho_{\epsilon}\left(y-x_{1}\right) \rho_{\epsilon}(t-s) d y d s d t \rightarrow \frac{1}{2} \int_{0}^{\tau} h_{1}^{-}(t) \eta\left(x_{1}, t\right) d t
\end{gathered}
$$
as $\epsilon \rightarrow 0^{+}$. Moreover, since $\iint_{Q_{1}} \rho_{\epsilon}\left(x_{1}-x\right) \rho_{\epsilon}(t-s) d x d s=\frac{1}{2}$ for $\epsilon<\min \{t, \tau-t\}$,

$$
\begin{aligned}
& \left|\int_{0}^{\tau} \iint_{Q_{1}} \varphi\left(u_{r}(x, t)\right) \eta\left(\frac{x+x_{1}}{2}, \frac{t+s}{2}\right) \rho_{\epsilon}\left(x_{1}-x\right) \rho_{\epsilon}(t-s) d x d t d s-\frac{1}{2} \int_{0}^{\tau} h_{1}^{-}(t) \eta\left(x_{1}, t\right) d t\right| \leq \\
& \quad \leq \iint_{Q_{1}} d x d s \rho_{\epsilon}\left(x_{1}-x\right) \rho_{\epsilon}(t-s) \int_{0}^{\tau} d t \varphi\left(u_{r}(x, t)\right)\left|\eta\left(\frac{x+x_{1}}{2}, \frac{t+s}{2}\right)-\eta\left(x_{1}, t\right)\right|+ \\
& \quad+\left|\int_{I_{1}} d x \rho_{\epsilon}\left(x_{1}-x\right) \int_{0}^{\tau}\left[\varphi\left(u_{r}(x, t)\right)-h_{1}^{-}(t)\right] \eta\left(x_{1}, t\right) d t \int_{0}^{\tau} \rho_{\epsilon}(t-s) d s\right| \leq \\
& \quad \leq \frac{\|\varphi\|_{L^{\infty}}}{2} \sup _{0 \leq x_{1}-x+|t-s| \leq \epsilon} \int_{0}^{\tau}\left|\eta\left(\frac{x_{1}+x}{2}, \frac{t+s}{2}\right)-\eta\left(x_{1}, t\right)\right| d t+ \\
& \quad+\left\|\rho_{1}\right\|_{\infty} \frac{1}{\epsilon} \int_{x_{1}-\epsilon}^{x_{1}}\left|\int_{0}^{\tau}\left[\varphi\left(u_{r}(x, t)\right)-h_{1}^{-}(t)\right] \eta\left(x_{1}, t\right) d t\right| d x .
\end{aligned}
$$


By the smoothness of $\eta$ and equality (4.6), the right-hand side of the above inequality vanishes as $\epsilon \rightarrow 0^{+}$. Therefore,

$$
\int_{0}^{\tau} \iint_{Q_{1}} \varphi\left(u_{r}(x, t)\right) \eta\left(\frac{x+x_{1}}{2}, \frac{t+s}{2}\right) \rho_{\epsilon}\left(x_{1}-x\right) \rho_{\epsilon}(t-s) d x d t d s \rightarrow \frac{1}{2} \int_{0}^{\tau} h_{1}^{-}(t) \eta\left(x_{1}, t\right) d t .
$$

It is similarly seen that

$$
\int_{0}^{\tau} \iint_{Q_{1}} \varphi\left(v_{r}(y, s)\right) \eta\left(\frac{x_{1}+y}{2}, \frac{t+s}{2}\right) \rho_{\epsilon}\left(y-x_{1}\right) \rho_{\epsilon}(t-s) d y d s d t \rightarrow \frac{1}{2} \int_{0}^{\tau} g_{1}^{-}(s) \eta\left(x_{1}, s\right) d s .
$$

Letting $\epsilon \rightarrow 0^{+}$in (5.7) we obtain that, for all $\eta \in C_{c}^{1}\left(\left(-\infty, x_{1}\right] \times(0, \tau)\right), \eta \geq 0$,

$$
\begin{aligned}
& \iint_{Q_{1}}\left\{\left|u_{r}(x, t)-v_{r}(x, t)\right| \eta_{t}(x, t)+\right. \\
+ & \left.\operatorname{sgn}\left(u_{r}(x, t)-v_{r}(x, t)\right)\left[\varphi\left(u_{r}(x, t)\right)-\varphi\left(v_{r}(x, t)\right)\right] \eta_{x}(x, t)\right\} d x d t \geq 0 .
\end{aligned}
$$

Now fix $t^{\prime}, t^{\prime \prime}$ such that $0<t^{\prime}<t^{\prime \prime}<\tau$, and $x_{0}<x_{1}$. Let $\alpha_{\delta}=\alpha_{\delta}(x)$ and $\beta_{\theta}=\beta_{\theta}(t)$ be two families of mollifiers, such that $0<\delta<1,0<\theta<\min \left\{t^{\prime}, \tau-t^{\prime \prime}\right\}$. Set in (5.8)

$$
\eta(x, t)=\eta_{\delta, \theta}(x, t):=\int_{t-t^{\prime \prime}}^{t-t^{\prime}} \beta_{\theta}(s) d s \int_{\left\|\varphi^{\prime}\right\|_{\infty}\left(t-t^{\prime \prime}\right)+x_{0}}^{x_{1}+\delta} \alpha_{\delta}(x-y) d y \quad(\delta>0),
$$

with $x \in\left(\left\|\varphi^{\prime}\right\|_{\infty}\left(t-t^{\prime \prime}\right)+x_{0}, x_{1}\right], t \in\left(t^{\prime}-\theta, t^{\prime \prime}+\theta\right)$ (clearly, $\eta_{\delta, \theta}$ is nonnegative and belongs to $\left.C_{c}^{\infty}\left(\left(-\infty, x_{1}\right] \times(0, \tau)\right)\right)$. Since $\alpha_{\delta}\left(x-x_{1}-\delta\right)=0$ if $x \in\left(-\infty, x_{1}\right]$,

$$
\begin{aligned}
& \iint_{Q_{1}}\left|u_{r}-v_{r}\right|\left[\beta_{\theta}\left(t-t^{\prime}\right)-\beta_{\theta}\left(t-t^{\prime \prime}\right)\right]\left(\int_{\left\|\varphi^{\prime}\right\|_{\infty}\left(t-t^{\prime \prime}\right)+x_{0}}^{x_{1}+\delta} \alpha_{\delta}(x-y) d y\right) d x d t- \\
- & \iint_{Q_{1}}\left\{\left\|\varphi^{\prime}\right\|_{\infty}\left|u_{r}-v_{r}\right|+\operatorname{sgn}\left(u_{r}-v_{r}\right)\left[\varphi\left(u_{r}\right)-\varphi\left(v_{r}\right)\right]\right\} \times \\
\times & \alpha_{\delta}\left(x-\left\|\varphi^{\prime}\right\|_{\infty}\left(t-t^{\prime \prime}\right)-x_{0}\right)\left(\int_{t-t^{\prime \prime}}^{t-t^{\prime}} \beta_{\theta}(s) d s\right) d x d t \geq 0 .
\end{aligned}
$$

Since $\left\|\varphi^{\prime}\right\|_{\infty}|u-v|+\operatorname{sgn}(u-v)[\varphi(u)-\varphi(v)] \geq 0$ for every $u, v \geq 0$, it follows that

$$
\iint_{Q_{1}}\left|u_{r}-v_{r}\right|\left[\beta_{\theta}\left(t-t^{\prime}\right)-\beta_{\theta}\left(t-t^{\prime \prime}\right)\right]\left(\int_{\left\|\varphi^{\prime}\right\|_{\infty}\left(t-t^{\prime \prime}\right)+x_{0}}^{x_{1}+\delta} \alpha_{\delta}(x-y) d y\right) d x d t \geq 0 .
$$

Let $\delta \rightarrow 0^{+}$in this inequality. Then, by the Dominated Convergence Theorem,

$$
\left.\int_{0}^{\tau} \int_{\left\|\varphi^{\prime}\right\|_{\infty}\left(t-t^{\prime \prime}\right)+x_{0}}^{x_{1}}\left|u_{r}(x, t)-v_{r}(x, t)\right|\left[\beta_{\theta}\left(t-t^{\prime}\right)-\beta_{\theta}\left(t-t^{\prime \prime}\right)\right]\right) d x d t \geq 0,
$$

whence as $\theta \rightarrow 0^{+}$

kruz2

$$
\int_{x_{0}}^{x_{1}}\left|u_{r}\left(x, t^{\prime \prime}\right)-v_{r}\left(x, t^{\prime \prime}\right)\right| d x \leq \int_{x_{0}-\left\|\varphi^{\prime}\right\|_{\infty}\left(t^{\prime \prime}-t^{\prime}\right)}^{x_{1}}\left|u_{r}\left(x, t^{\prime}\right)-v_{r}\left(x, t^{\prime}\right)\right| d x .
$$

Since $u, v \in C\left([0, T] ; \mathcal{M}^{+}(\Omega)\right)$, letting $t^{\prime} \rightarrow 0^{+}$in (5.9) we obtain for all $\left(x_{0}, t^{\prime \prime}\right) \in Q_{1}$

$$
\int_{x_{0}}^{x_{1}}\left|u_{r}\left(x, t^{\prime \prime}\right)-v_{r}\left(x, t^{\prime \prime}\right)\right| d x \leq \int_{x_{0}-\left\|\varphi^{\prime}\right\|_{\infty} t^{\prime \prime}}^{x_{1}}\left|u_{r}(x, 0)-v_{r}(x, 0)\right| d x=0,
$$

since $u_{r}(\cdot, 0)=v_{r}(\cdot, 0)=u_{0 r}$. Since $t^{\prime \prime} \in(0, \tau)$ is arbitrary, it follows that $u_{r}=v_{r}$ in $Q_{1}$. 
To prove Theorem 3.2 we need the following result.

curd Lemma 6.1. Let $\left(H_{1}\right)$ be satisfied. Let $v_{0} \in \mathcal{M}^{+}(\mathbb{R})$ satisfy $\left(H_{0}\right)$, and let $u_{0} \leq v_{0}$ in $\mathcal{M}(\mathbb{R})$. Let $u, v \in C\left([0, T] ; \mathcal{M}^{+}(\mathbb{R})\right)$ be the unique entropy solutions of $(P)$ with initial data $u_{0}, v_{0}$ given by Theorem 3.1. Set

$$
\tau:=\min \left\{t_{u}, t_{v}\right\} \quad \text { where } \quad\left\{\begin{array}{l}
t_{u}:=\sup \left\{t \in[0, T) \mid \operatorname{supp} u_{s}(\cdot, t)=\operatorname{supp} u_{0 s}\right\}, \\
t_{v}:=\sup \left\{t \in[0, T) \mid \operatorname{supp} v_{s}(\cdot, t)=\operatorname{supp} v_{0 s}\right\} .
\end{array}\right.
$$

Then

disfobis (6.2)

$$
u_{r}(\cdot, t) \leq v_{r}(\cdot, t) \quad \text { a.e. in } \mathbb{R} \text { for any } t \in[0, \tau] .
$$

Proof. By assumption there holds $u_{0 s}=\sum_{j=1}^{p} c_{j} \delta_{x_{j}}, v_{0 s}=\sum_{j=1}^{p} d_{j} \delta_{x_{j}}$ with

$$
0 \leq c_{j} \leq d_{j}, \quad d_{j}>0 \text { for all } j=1, \ldots, p .
$$

Suppose first $c_{j}>0$ for all $j=1, \ldots, p$. Let $u_{1, n}$ and $v_{1, n}$ be the entropy solutions of problem $\left(P_{1, n}\right)$ with initial data $u_{0 n}:=\min \left\{u_{0 r}, n\right\}$ and $v_{0 n}:=\min \left\{v_{0 r}, n\right\}$, respectively. From inequality (4.22) we get for any $\left(x_{0}, t\right) \in I_{1} \times[0, \tau)$

$$
\int_{x_{0}}^{x_{1}}\left[u_{1, n}(x, t)-v_{1, n}(x, t)\right]_{+} d x \leq \int_{x_{0}-\left\|\varphi^{\prime}\right\|_{\infty} t}^{x_{1}}\left[u_{0 n}(x)-u_{0 n}(x)\right]_{+} d x .
$$

Since, by uniqueness and the proof of Theorem 4.1 (see (4.25) and (4.35) ), $u_{1, n} \rightarrow u_{r}$ and $v_{1, n} \rightarrow v_{r}$ a.e. in $S_{1}$, we obtain from Fatou's Lemma that

$$
\int_{x_{0}}^{x_{1}}\left[u_{r}(x, t)-v(x, t)\right]_{+} d x \leq \int_{x_{0}-\left\|\varphi^{\prime}\right\|_{\infty} t}^{x_{1}}\left[u_{0 r}(x)-v_{0 r}(x)\right]_{+} d x
$$

for every $t \in(0, \tau)$. Similar inequalities can be proven in $I_{j} \times[0, \tau)$ for $j=2, \ldots, p+1$, thus for every $x^{\prime}, x^{\prime \prime} \in \mathbb{R}, x^{\prime}<x^{\prime \prime}$, and $t \in[0, \tau]$

$$
\int_{x^{\prime}}^{x^{\prime \prime}}\left[u_{r}(x, t)-v_{r}(x, t)\right]_{+} d x \leq \int_{x^{\prime}-\left\|\varphi^{\prime}\right\|_{\infty} t}^{x^{\prime \prime}+\left\|\varphi^{\prime}\right\|_{\infty} t}\left[u_{0 r}(x)-v_{0 r}(x)\right]_{+} d x .
$$

Hence the result follows in this case.

Now let $c_{k}=0$ for some $k \in\{1, \ldots, p\}$; we only give the proof when $c_{1}=0$ and $c_{j}>0$ for $j=2, \ldots, p$, since the general case is similar. Consider two sequences $\left\{u_{0 m}\right\},\left\{v_{0 m}\right\} \subset B V_{l o c}\left(\bar{I}_{1} \cup I_{2}\right)$ such that $u_{0 m} \rightarrow u_{0 r}, v_{0 m} \rightarrow v_{0 r}$ in $L_{l o c}^{1}\left(\bar{I}_{1} \cup I_{2}\right)$ as $m \rightarrow \infty$, and $u_{0 m} \leq v_{0 m}$ a.e. in $\bar{I}_{1} \cup I_{2}$ for all $m \in \mathbb{N}$. Set $u_{0 m, n}:=\min \left\{u_{0 m}, n\right\}$, $v_{0 m, n}:=\min \left\{v_{0 m}, n\right\}(n \in \mathbb{N})$, then consider the problems

$$
\begin{gathered}
\begin{cases}u_{t}+[\varphi(u)]_{x}=0 & \text { in }\left(\bar{I}_{1} \cup I_{2}\right) \times(0, \tau) \\
u=n & \text { in } \partial\left(\bar{I}_{1} \cup I_{2}\right) \times(0, \tau) \\
u=u_{0 m, n} & \text { in }\left(\bar{I}_{1} \cup I_{2}\right) \times\{0\},\end{cases} \\
\left\{\begin{array}{ll}
v_{t}+[\varphi(v)]_{x}=0 & \text { in } I_{i} \times(0, \tau) \\
v=n & \text { in } \partial I_{i} \times(0, \tau) \\
v=v_{0 m, n} & \text { in } I_{i} \times\{0\}
\end{array} \quad(i=1,2) .\right.
\end{gathered}
$$

Let $u_{m, n} \in B V_{l o c}\left(\left(\bar{I}_{1} \cup I_{2}\right) \times(0, \tau)\right)$ and $v_{i m, n} \in B V_{l o c}\left(I_{i} \times(0, \tau)\right)$ be the unique entropy solution of $(U)$ and $\left(V_{i}\right)$, respectively. Since $u_{m, n} \in B V_{l o c}\left(\left(\bar{I}_{1} \cup I_{2}\right) \times\right.$ $(0, \tau))$, for a.e. $t \in(0, \tau)$ there exist the traces $u_{m, n}\left(x_{1}^{ \pm}, t\right)$; moreover, there holds 
$u_{m, n}\left(x_{1}^{ \pm}, \cdot\right) \leq n$ by comparison results (see the proof of Lemma 4.6). Then, since $u_{0 m, n} \leq v_{0 m, n}$ a.e. in $\bar{I}_{1} \cup I_{2}$, it follows easily by comparison that

$$
u_{m, n} \leq v_{i m, n} \text { a.e. in } I_{i} \times(0, \tau) \quad(i=1,2) .
$$

As $m \rightarrow \infty$ there holds $u_{0 m, n} \rightarrow u_{0 n}, v_{0 m, n} \rightarrow v_{0 n}$ in $L_{l o c}^{1}\left(\bar{I}_{1} \cup I_{2}\right)$. Then $u_{m, n}$ converges in $L_{l o c}^{1}\left(\left(\bar{I}_{1} \cup I_{2}\right) \times(0, \tau)\right)$ to the unique entropy solution $u_{n}$ of the problem

$$
\begin{cases}u_{t}+[\varphi(u)]_{x}=0 & \text { in }\left(\bar{I}_{1} \cup I_{2}\right) \times(0, \tau) \\ u=n & \text { in } \partial\left(\bar{I}_{1} \cup I_{2}\right) \times(0, \tau) \\ u=u_{0 n} & \text { in }\left(\bar{I}_{1} \cup I_{2}\right) \times\{0\}\end{cases}
$$

(see 9]). Similarly, $v_{i m, n}$ converges in $L_{l o c}^{1}\left(\left(I_{i}\right) \times(0, \tau)\right)$ to the unique entropy solution $v_{\text {in }}$ of the problem

$$
\left\{\begin{array}{ll}
v_{t}+[\varphi(v)]_{x}=0 & \text { in } I_{i} \times(0, \tau) \\
v=n & \text { in } \partial I_{i} \times(0, \tau) \\
v=v_{0 n} & \text { in } I_{i} \times\{0\}
\end{array} \quad(i=1,2) .\right.
$$

Then letting $m \rightarrow \infty$ in (6.4) (possibly up to subsequences) we get

$$
u_{n} \leq v_{i n} \text { a.e. in } I_{i} \times(0, \tau) \quad(i=1,2) .
$$

By uniqueness and the proof of Theorem 4.1, as $n \rightarrow \infty$ there holds $u_{n} \rightarrow u_{r}$ a.e. in $\left(\bar{I}_{1} \cup I_{2}\right) \times(0, \tau), v_{i n} \rightarrow v_{r}$ a.e. in $I_{i} \times(0, \tau)$ for $i=1,2$. Then from (6.5) letting $n \rightarrow \infty$ we obtain that $u_{r} \leq v_{r}$ a.e. in $\left(\bar{I}_{1} \cup I_{2}\right) \times(0, \tau)$. It is similarly seen that $u_{r} \leq v_{r}$ a.e. in $\bigcup_{j=3}^{p+1} I_{j} \times(0, \tau)$; since $u_{r}, v_{r} \in C\left([0, T] ; L_{l o c}^{1}(\mathbb{R})\right)$, the result follows.

Proof of Theorem 3.2. By (6.2) there holds

$$
u_{a c}(\cdot, t) \leq v_{a c}(\cdot, t) \quad \text { in } \mathcal{M}(\mathbb{R}) \quad \text { for any } t \in[0, \tau],
$$

with $\tau$ given by (6.1). Let us prove that

$$
u_{s}(\cdot, t) \leq v_{s}(\cdot, t) \quad \text { in } \mathcal{M}(\mathbb{R}) \quad \text { for any } t \in[0, \tau] .
$$

We only prove (6.7) when every $c_{j}$ is positive, since the proof is similar (and easier) if some $c_{j}$ is zero.

To this purpose, as in the proof of Theorem [5.1] we set $Q_{1}:=\left(-\infty, x_{1}\right] \times[0, \tau)$ and apply Kružkov's method with $\xi=\xi(x, t, y, s)$ defined in $Q_{1} \times Q_{1}$, such that $\xi(\cdot, \cdot, y, s) \in C_{c}^{1}\left(Q_{1}\right)$ for every $(y, s) \in Q_{1}$ and $\xi(x, t, \cdot, \cdot) \in C_{c}^{1}\left(Q_{1}\right)$ for every $(x, t) \epsilon$ $Q_{1}$. From (4.13)-(4.14) we get

$$
\begin{aligned}
& \iint_{Q_{1}}\left\{H_{+}\left(u_{r}(x, t)-v_{r}(y, s)\right)\left[\varphi\left(u_{r}(x, t)\right)-\varphi\left(v_{r}(y, s)\right)\right] \xi_{x}(x, t, y, s)+\right. \\
& \left.+\left[u_{r}(x, t)-v_{r}(y, s)\right]_{+} \xi_{t}(x, t, y, s)\right\} d x d t \geq \int_{0}^{\tau}\left[h_{1}^{-}(t)-\varphi\left(v_{r}(y, s)\right)\right] \xi\left(x_{1}, t, y, s\right) d t \\
& \iint_{Q_{1}}\left\{H_{-}\left(v_{r}(y, s)-u_{r}(x, t)\right)\left[\varphi\left(v_{r}(y, s)\right)-\varphi\left(u_{r}(x, t)\right)\right] \xi_{y}(x, t, y, s)+\right. \\
& \left.+\left[v_{r}(y, s)-u_{r}(x, t)\right]_{-} \xi_{s}(x, t, y, s)\right\} d x d t=\iint_{Q_{1}}\left\{\left[u_{r}(x, t)-v_{r}(y, s)\right]_{+} \xi_{s}(x, t, y, s)+\right. \\
& \left.+H_{+}\left(u_{r}(x, t)-v_{r}(y, s)\right)\left[\varphi\left(u_{r}(x, t)\right)-\varphi\left(v_{r}(y, s)\right)\right] \xi_{y}(x, t, y, s)\right\} d x d t \geq 0 .
\end{aligned}
$$


Choosing $\xi$ as in (5.6), from the above inequalities we get

$$
\begin{aligned}
& \iiint \int_{Q_{1} \times Q_{1}} \rho_{\epsilon}(x-y) \rho_{\epsilon}(t-s)\left\{\left[u_{r}(x, t)-v_{r}(y, s)\right]_{+} \eta_{t}\left(\frac{x+y}{2}, \frac{t+s}{2}\right)+\right. \\
& \left.+H_{+}\left(u_{r}(x, t)-v_{r}(y, s)\right)\left[\varphi\left(u_{r}(x, t)\right)-\varphi\left(v_{r}(y, s)\right)\right] \eta_{x}\left(\frac{x+y}{2}, \frac{t+s}{2}\right)\right\} d x d t d y d s \geq \\
& \geq \int_{0}^{\tau} \iint_{Q_{1}}\left[h_{1}^{-}(t)-\varphi\left(v_{r}(y, s)\right)\right] \eta\left(\frac{x_{1}+y}{2}, \frac{t+s}{2}\right) \rho_{\epsilon}\left(y-x_{1}\right) \rho_{\epsilon}(t-s) d y d s d t .
\end{aligned}
$$

Then arguing as in the proof of Theorem 5.1plainly gives

$$
\begin{aligned}
& \iint_{Q_{1}}\left\{H_{+}\left(u_{r}(x, t)-v_{r}(x, t)\right)\left[\varphi\left(u_{r}(x, t)\right)-\varphi\left(v_{r}(x, t)\right)\right] \eta_{x}(x, t)+\right. \\
& \left.+\left[u_{r}(x, t)-v_{r}(x, t)\right]_{+} \eta_{t}(x, t)\right\} d x d t \geq \frac{1}{2} \int_{0}^{\tau}\left[h_{1}^{-}(t)-g_{1}^{-}(t)\right] \eta\left(x_{1}, t\right) d t
\end{aligned}
$$

for every $\eta \in C_{c}^{1}\left(\left(-\infty, x_{1}\right] \times(0, \tau)\right), \eta \geq 0$, with $h_{1}^{-}, g_{1}^{-}$given by (4.4) and (5.5), respectively.

From (6.2) and (6.8) we obtain

$$
h_{1}^{-} \leq g_{1}^{-} \quad \text { a.e in }(0, \tau) .
$$

It is similarly proven that (see Remark 4.3):

$\operatorname{cflu}$

$$
h_{j}^{-} \leq g_{j}^{-} \text {for } j=2, \ldots, p, \quad h_{j}^{+} \geq g_{j}^{+} \text {for } j=1, \ldots, p \quad \text { a.e. in }(0, \tau) .
$$

Now observe that, by uniqueness and the proof of Theorem 4.1

$$
u_{s}(\cdot, t)=\sum_{j=1}^{p} C_{j}(t) \delta_{x_{j}}, \quad v_{s}(\cdot, t)=\sum_{j=1}^{p} D_{j}(t) \delta_{x_{j}},
$$

with $C_{j}$ defined by (4.34) and

$$
D_{j}(t):=\left[d_{j}-\int_{0}^{t}\left[g_{j}^{+}(s)-g_{j}^{-}(s)\right] d s\right]_{+} \quad(j=1, \ldots, p) .
$$

By (6.3) and (6.10) there holds $C_{j}(t) \leq D_{j}(t)$ for all $j=1, \ldots, p$ and $t \in[0, \tau]$, thus inequality (6.7) follows.

Hence there holds $u(\cdot, t) \leq v(\cdot, t)$ in $\mathcal{M}(\mathbb{R})$ for all $t \in[0, \tau]$. Arguing as at the end of the proof of Theorem 4.1 we obtain the conclusion.

Remark 6.1. In section 5 we used Kružkov's method to prove the uniqueness of entropy solutions satisfying the compatibility conditions. In the above proof we used the same method to compare the fluxes of two such solutions at points where their singular parts are nontrivial and their regular parts are locally ordered. This additional information is contained in (6.9) $-(6.10)$.

\section{REFERENCES}

[1] L. Ambrosio, N. Fusco \& D. Pallara, Functions of Bounded Variation and Free Discontinuity Problems, (Oxford University Press, 2000).

[2] C. Bardos, A. Y. Le Roux \& J. C. Nedelec, First order quasilinear equations with boundary condition, Comm. Partial Differential Equations 4 (1979), 1017-1034.

[3] M. Bertsch, F. Smarrazzo, A. Terracina \& A. Tesei, Radon measure-valued solutions of first order hyperbolic conservation laws, preprint (2017).

[4] G.-Q. Chen \& Bo Su, Discontinuous solutions of Hamilton-Jacobi equations: Existence, uniqueness, and regularity, Hyperbolic Problems: Theory, Numerics, Applications, T.Y. Hou et al. Eds. (Springer, 2003).

[5] F. Demengel \& D. Serre, Nonvanishing singular parts of measure valued solutions for scalar hyperbolic equations, Comm. Part. Diff. Equ. 16 (1991), 221-254. 
[6] L.C. Evans, Envelopes and nonconvex Hamilton-Jacobi equations, Calc. Var \& PDE 50 (2014), 257-282.

[7] A. Friedman, Mathematics in Industrial Problems, Part 8, IMA Volumes in Mathematics and its Applications 83 (Springer, 1997).

[8] T.-P. Liu \& M. Pierre, Source-solutions and asymptotic behavior in conservation laws, J. Differential Equations 51 (1984), 419-441.

[9] J. Málek, J. Nečas, M. Rokyta \& M. Rúžička, Weak and Measure-valued Solutions of Evolutionary PDEs (Chapman \& Hall, 1996).

[10] F. Otto, Initial-boundary value problem for a scalar conservation law, Comptes Rendus Acad. Sci. 322 (1996), 729-734.

[11] D.S. Ross, Two new moving boundary problems for scalar conservation laws, Comm. Pure Appl. Math 41 (1988), 725-737.

[12] D.S. Ross, Ion etching: An application of the mathematical theory of hyperbolic conservation laws, J. Electrochem. Soc. 135 (1988), 1235-1240.

[13] D. Serre, Systems of Conservation Laws, Vol. 1: Hyperbolicity, Entropies, Shock Waves, (Cambridge University Press, 1999).

[14] A. Terracina, Comparison properties for scalar conservation laws with boundary conditions, Nonlinear Anal. 28 (1997), 633- 653.

Dipartimento di Matematica, Universitì di Roma Tor Vergata, Via della Ricerca Scientifica, 00133 Roma, Italy, and Istituto per le Applicazioni del Calcolo "M. Picone", CNR, ROMA, ITALY

E-mail address: bertsch.michiel@gmail.com

Università Campus Bio-Medico di Roma, Via Alvaro del Portillo 21, 00128 Roma, ITALY

E-mail address: flavia.smarrazzo@gmail.com

Dipartimento di Matematica "G. Castelnuovo", Università Sapienza di Roma, P.le A. Moro 5, I-00185 Roma, ItAly

E-mail address: terracina@mat.uniroma1.it

Dipartimento di Matematica "G. Castelnuovo", Università Sapienza di Roma, P.le A. Moro 5, I-00185 Roma, Italy, and Istituto per le Applicazioni del Calcolo "M. Picone", CNR, ROMA, ITALY

E-mail address: albertotesei@gmail.com 